\title{
Distributed Smart Grid Asset Control Strategies for Providing Ancillary Services
}

K Kalsi

J Lian

C Moya
W Zhang

LD Marinovici

JE Dagle

September 2013

Pacific Northwest

NATIONAL LABORATORY

Proudly Operated by Battelle Since 1965 


\title{
DISCLAIMER
}

This report was prepared as an account of work sponsored by an agency of the United States Government. Neither the United States Government nor any agency thereof, nor Battelle Memorial Institute, nor any of their employees, makes any warranty, express or implied, or assumes any legal liability or responsibility for the accuracy, completeness, or usefulness of any information, apparatus, product, or process disclosed, or represents that its use would not infringe privately owned rights. Reference herein to any specific commercial product, process, or service by trade name, trademark, manufacturer, or otherwise does not necessarily constitute or imply its endorsement, recommendation, or favoring by the United States Government or any agency thereof, or Battelle Memorial Institute. The views and opinions of authors expressed herein do not necessarily state or reflect those of the United States Government or any agency thereof.

\author{
PACIFIC NORTHWEST NATIONAL LABORATORY \\ operated by \\ BATTELLE \\ for the
}

\section{UNITED STATES DEPARTMENT OF ENERGY}

under Contract DE-AC05-76RL01830

\section{Printed in the United States of America}

Available to DOE and DOE contractors from the Office of Scientific and Technical Information,

P.O. Box 62, Oak Ridge, TN 37831-0062; ph: (865) 576-8401 fax: (865) 576-5728 email: reports@adonis.osti.gov

Available to the public from the National Technical Information Service, U.S. Department of Commerce, 5285 Port Royal Rd., Springfield, VA 22161 ph: (800) 553-6847 fax: (703) 605-6900 email: orders@ntis.fedworld.gov online ordering: http://www.ntis.gov/ordering.htm This document was printed on recycled paper. 


\title{
Distributed Smart Grid Asset Control Strategies for Providing Ancillary Services
}

\author{
K Kalsi \\ W Zhang \\ J Lian \\ LD Marinovici \\ C Moya \\ JE Dagle
}

September 2013

Prepared for

the U.S. Department of Energy

under Contract DE-AC05-76RL01830

Pacific Northwest National Laboratory

Richland, Washington 99352 



\section{Executive summary}

With large-scale plans to integrate renewable generation driven mainly by state-level renewable portfolio requirements, more resources will be needed to compensate for the uncertainty and variability associated with intermittent generation resources. Distributed assets can be used to mitigate the concerns associated with renewable energy resources and to keep costs down. Under such conditions, performing primary frequency control using only supply-side resources becomes not only prohibitively expensive but also technically difficult. It is therefore important to explore how a sufficient proportion of the loads could assume a routine role in primary frequency control to maintain the stability of the system at an acceptable cost.

The main objective of this project is to develop a novel hierarchical distributed framework for frequency based load control. The framework involves two decision layers. The top decision layer determines the optimal gain for aggregated loads for each load bus. The gains are computed using decentralized robust control methods, and will be broadcast to the corresponding participating loads every control period. The second layer consists of a large number of heterogeneous devices, which switch probabilistically during contingencies so that aggregated power change matches the desired amount according to the most recently received gains. The simulation results show great potential to enable systematic design of demand-side primary frequency control with stability guarantees on the overall power system. The proposed design systematically accounts for the interactions between the total load response and bulk power system frequency dynamics. It also guarantees frequency stability under a wide range of time varying operating conditions. The local device-level load response rules fully respect the device constraints (such as temperature setpoint, compressor time delays of HVACs, or arrival and departure of the deferrable loads), which are crucial for implementing real load control programs.

The promise of autonomous, Grid Friendly ${ }^{\mathrm{TM}}$ response by smart appliances in the form of underfrequency load shedding was demonstrated in the GridWise Olympic Peninsula Demonstration in 2006. Each controller monitored the power grid voltage signal and requested that electrical load be shed by its appliance whenever electric power-grid frequency fell below $59.95 \mathrm{~Hz}$. The controllers and their appliances responded reliably to each shallow under-frequency event, which was an average of one event per day and shed their loads for the durations of these events. Another objective of this project was to perform extensive simulation studies to investigate the impact of a population of Grid Friendly ${ }^{\mathrm{TM}}$ Appliances (GFAs) on the bulk power system frequency stability. The GFAs considered in this report are represented as demonstration units with water heaters individually modeled. 



\section{Acknowledgments}

The authors are grateful for the comments and feedback provided by Jason Fuller, David Chassin and Don Hammerstrom at the Pacific Northwest National Laboratory. 



\section{Acronyms and Abbreviations}

LMIs

GFAs

TCL

PSLF

HVAC

PST
Linear Matrix Inequalities

Grid Friendly ${ }^{\mathrm{TM}}$ Appliances

Thermostatically Controlled Loads

Positive Sequence Load Flow

Heating, Ventilation, and Air Conditioning

Power System Toolbox 



\section{Contents}

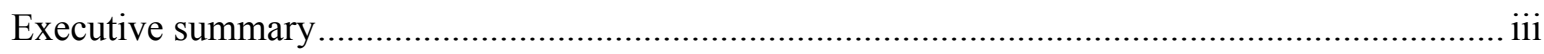

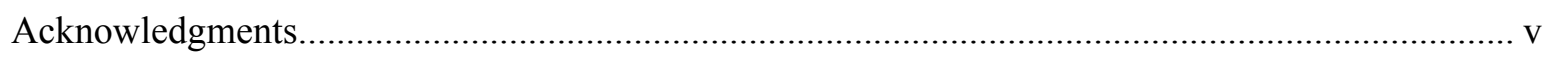

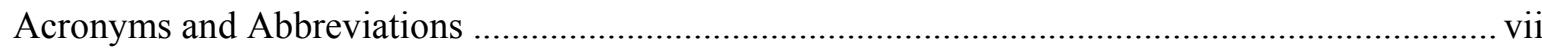

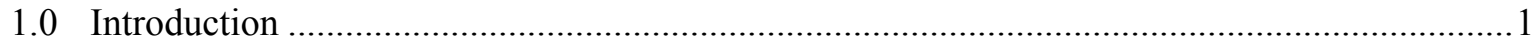

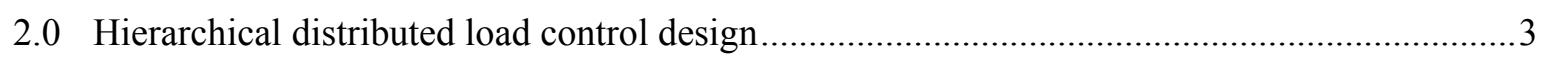

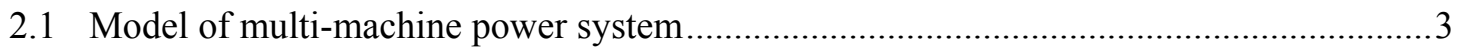

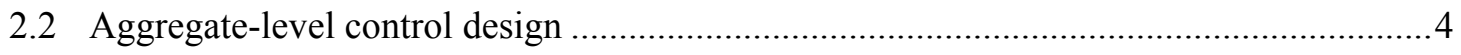

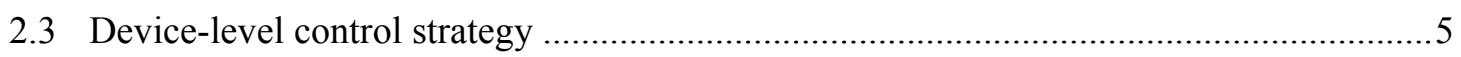

2.4 Simulations studies for proposed load control design ................................................

2.4.1 Validation over a IEEE 13-bus 4-generators system.......................................... 8

2.4.2 Validation over a IEEE 68-bus 16-generators system........................................ 13

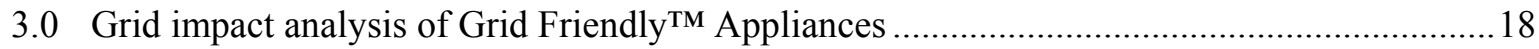

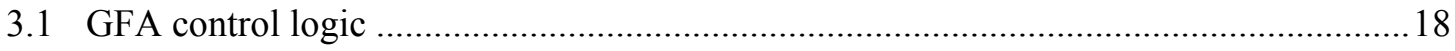

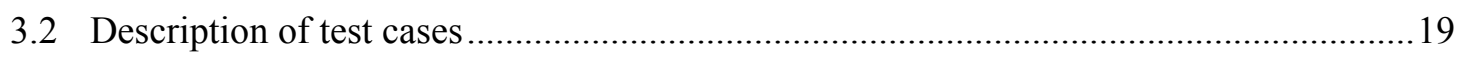

3.3 Power System Toolbox implementation of the GFA control logic .................................20

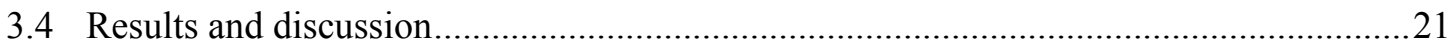

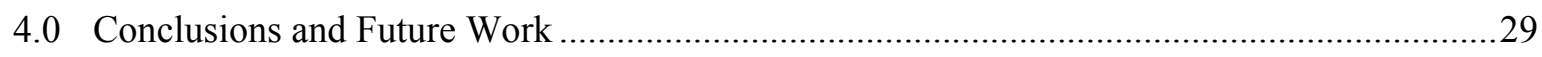

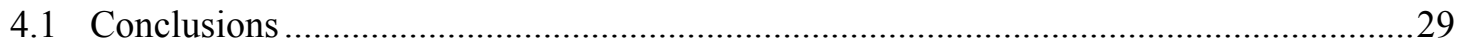

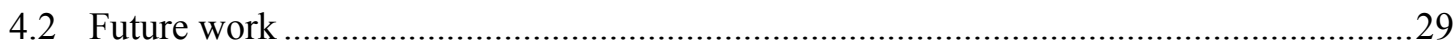

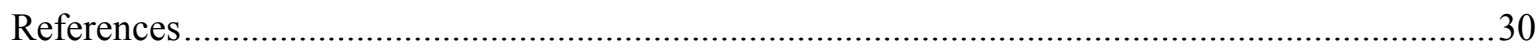




\section{Figures}

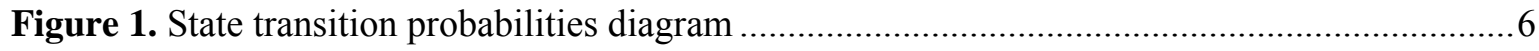

Figure 2. IEEE 2-area, 4-generators, 13-bus power system ........................................................

Figure 3. Frequency response with only generation side droop control ..................................... 9

Figure 4. Frequency response at load bus-4 (simplified version of controller presented in [1]) .....10

Figure 5. Active power consumption at load bus-4 (simplified version of controller presented in

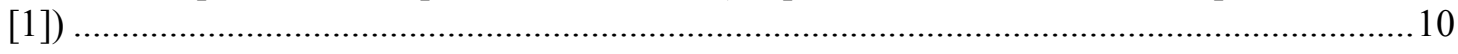

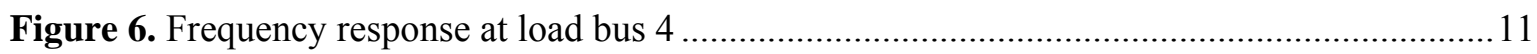

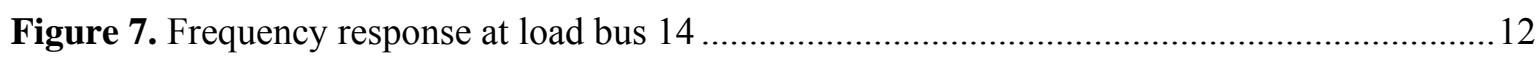

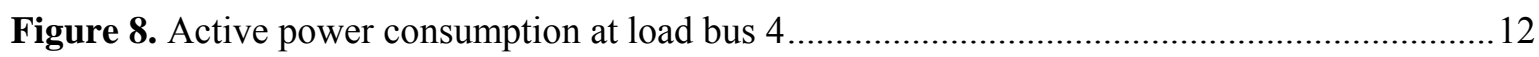

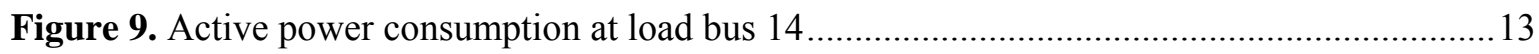

Figure 10. IEEE 16-machine, 86-transmission line, 68-bus power system (adapted from [10]) .....14

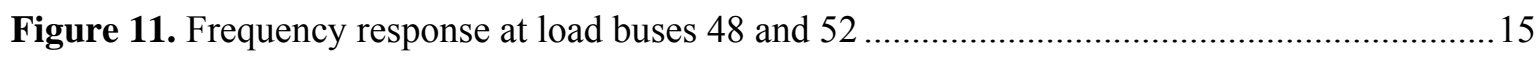

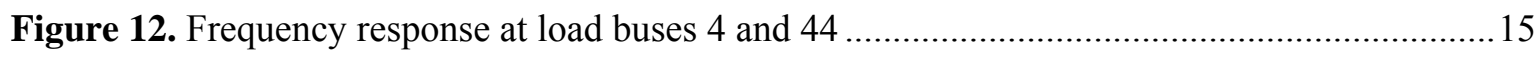

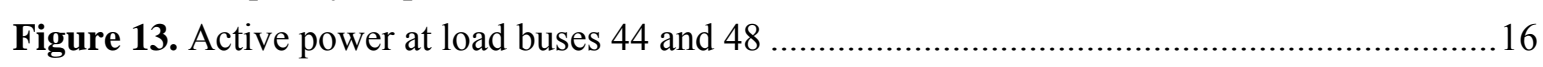

Figure 14. Frequency response at load bus 4 (Small vs. large feedback gains) .............................17

Figure 15. Active power consumption at load bus 4 (Small vs. large feedback gains)..................17

Figure 16. Underfrequency control logic for an individual water heater with GFA controller .......19

Figure 18. Generator frequency response under GFA control with 0.4 second response time ........22

Figure 19. Bus active power under GFA control with 0.4 second response time .........................23

Figure 20. Generator frequency response under GFA control with different response times..........24

Figure 21. Generator frequency response under GFA controller with 0.4 second response time....25

Figure 22. Generator frequency response under GFA controller with 0.2 second response time....26

Figure 23. Generator frequency response under GFA controller with 0.6 second response time....27

Figure 24. Generator frequency response under different penetration of GFA loads ....................28 


\subsection{Introduction}

Frequency control plays an important role in preserving the power balance of a multi-machine power system. Generators modify their power output when a non-zero frequency deviation is presented in order to restore power balance across the network. However, with plans for large-scale penetration of renewable energy resources, performing primary frequency control using only supply-side resources becomes not only prohibitively expensive but also technically difficult. Frequency control from the demand side or load control presents a novel and viable way for providing the desired frequency response. Loads can measure frequency locally and change their power consumption after a non-zero frequency deviation is presented in order to achieve power balance between generation and consumption.

Several load control implementations have been proposed in the literature. In [1], it was shown how demand-side resources can contribute to primary frequency control without requiring explicit communication. The authors in [2] developed frequency-based load control strategies in order to balance demand with supply, and regulate frequency in power systems. Most of these works were developed using a reduced power system modeled as an infinite bus (only one bus); which is clearly not suitable for a decentralized and systematic implementation. One of the first attempts to study load control on a more general multi-machine power system model to achieve power balance while minimizing end user disutility was presented in [3]. Other practical issues have been studied in order to develop a fast load control with stochastic frequency measurement errors as shown in [4]. The approach seeks to match the real-time demand with supply and regulate the frequency while minimizing the global end-use dis-utility with a small amount of communication between loads.

Due to the nature of the power system dynamics coupled with controllable loads, Linear Matrix Inequalities (LMIs) constitute a great tool to develop a systematic and decentralized load control law that balances power across the network. The design of LMI based control strategies has been studied extensively in [6]. Particularly, the robust stabilization of nonlinear systems using LMIs was presented in [7], and later applied to power systems as shown in [8]-[11]. Moreover, notice that load control requires that an extensive population of controllable loads respond accordingly to achieve the desired power balance across the network. Thus, aggregated models for a heterogeneous population of controllable loads such as thermostatic controlled loads (HVAC's, refrigerators, water heaters, etc.) are necessary to accurately capture their collective behavior. These aggregated models have been studied in [12], [14] and [15]. Moreover, practical constraints such us compressor lockout have been incorporated in the aggregate models, to capture their dynamics in more realistic environments [12].

The specific outcomes of this project were to:

- Develop a framework to facilitate large-scale deployment of frequency responsive end-use devices

- Provide a systematic design methodology of decentralized frequency-based load control strategies for enhanced stability performance

- Ensure applicability of the control strategy over wide range of operating conditions while accounting for unpredictable end-use behavior and physical device constraints 
- Create a level-playing field for smart grid assets with conventional generators

- Enable high penetration of renewable energy resources

In this project, hierarchical decentralized load control strategies using linear matrix inequalities were proposed while accounting for several practical considerations from a demand side point of view. The proposed control strategy was tested on a multi-machine power system model. A simple aggregated model for responsive loads was developed in order to point out the different practical issues that have to be taken into account for the implementation of the feedback load control law. The complete architecture is able to accurately reestablish power balance on the proposed test system subject to structural perturbations such as contingencies and other system disturbances. The main novelty of the proposed approach lies in its decentralized load controller design with a systematic consideration of the impact of the demand-side primary frequency response on the overall power system frequency dynamics. 


\subsection{Hierarchical distributed load control design}

In this section, a systematic way of designing hierarchical distributed load control for frequency stabilization is proposed. At the aggregate level, optimal load control gains are derived for each load bus based on the decentralized robust control theory. The optimal control gains for each load bus will be used to specify the power modulation command for controllable loads at that bus in order to stabilize the power system during contingency. At the device level at each load bus, decentralized controllers are designed for each individual device based on Markov Chains so that the change of power consumption of controllable loads matches the desired power modulation command determined at the aggregate level.

\subsection{Model of multi-machine power system}

The design of the proposed hierarchical distributed load control strategy requires a multi-machine power system model for the stability analysis. Consider the following $n$ machine classic model,

$$
\begin{aligned}
& \dot{\theta}_{i}=\omega_{i} \\
& M_{i} \dot{\omega}_{i}=P_{m i}-P_{e i}
\end{aligned}
$$

where $\theta_{i}$ is the $i$-th machine angle, $\omega_{i}$ is the $i$-th machine frequency, $M_{i}$ is the $i$-th machine inertia, $P_{m_{i}}$ is the $i$-th machine mechanical power input, and $P_{e_{i}}$ is the $i$-th machine electrical power output. The power difference $P_{m_{i}}-P_{e_{i}}$ represents the power imbalance for the $i$-th generator. The power system is said to be synchronized, i.e. all the generators are at nominal frequency. For the model considered in this study, the mechanical power input $P_{m_{i}}$ is set to be constant. The electrical power output of the generator has the following mathematical form,

$$
P_{e i}=D_{G i} \omega_{i}+D_{L i} \omega_{i}+P_{i}+f(\theta)
$$

where the term $D_{G_{i}}$ is the mechanical damping of the $i$-th machine, $D_{L_{i}}$ is the load damping from the frequency sensitive loads [16] and [17], $P_{i}$ represents the load power consumption at the bus, and finally, the term $f(\theta)$ represents the power flow between buses. The term $f(\theta)$ simply represents the interactions between interconnected buses and loses across the network. Most of the previous works in demand-side frequency control have neglected or simplified this interaction by assuming an infinite bus model or a lossless power transmission network. Notice that, by substituting (2) into (1), the power system dynamics can be expressed in the following detailed mathematical form,

$$
\begin{aligned}
& M_{i} \ddot{\theta}_{i}=-D_{i} \dot{\theta}_{i}+P_{m, i}-P_{i}-\sum_{j \neq i} P_{i j} \sin \left(\theta_{i}-\theta_{j}+\varphi_{i j}\right) \\
& P_{i}=P_{i}^{o}+u_{i}\left(\theta_{i}, \dot{\theta}_{i}\right)
\end{aligned}
$$

where $D_{i}=D_{G_{i}}+D_{L i}, P_{i}^{o}$ is the nominal load power consumption of the loads (controllable and noncontrollable) when the controllable loads are not modulated, and $u_{i}$ is the power modulation command for 
controllable loads which will be derived later in this report. Moreover, $P_{i j}=\left|V_{i}\right|\left|V_{j}\right| Y_{i j} \mid>0$ is the maximum power transferred from generator bus $i$ to generator bus $j$, with $\left|V_{k}\right|$ as the regulated voltage at any bus $\mathrm{k}$ and $\left|Y_{i j}\right|$ as the admittance of the transmission line between bus $i$ and bus $j$. Additionally,

$\varphi_{i j}=\frac{\Re\left(Y_{i j}\right)}{\mathfrak{I}\left(Y_{i j}\right)}$ reflects the loses of the network with the susceptance given by $\Re\left(Y_{i j}\right)$ and the conductance given by $\mathfrak{I}\left(Y_{i j}\right)$.

\subsection{Aggregate-level control design}

To design the controller $u_{i}$ for power modulation command, a network-reduced model of a multimachine power system was considered, where load buses are suppressed and combined with nearby generator buses. Every $T$ minutes, the number of available controllable loads and their operating conditions are determined. Next, the load power modulation command $u_{i}$ is derived by formulating and solving a Linear Matrix Inequality (LMI) based optimization problem. This is an approach commonly used in decentralized robust control theory to ensure feasibility over a wide range of operating conditions. For the implementation of the LMI based optimization approach, the system needs to be a complete dynamical interconnected system as described in [7] and [8]. In particular, from a control theoretic point of view, each bus can be considered as an interconnected subsystem which is stabilizable or input reachable. This implies that if there exists a non-zero deviation of the frequency after any structural perturbation, under the assumption that sufficient controllable power is available from the demand side, the load power modulation can reestablish power balance.

A detailed derivation of the controller, using LMIs is presented next. The states defined before are clustered in the following vector: $x_{i}=\left[\theta_{i}, \omega_{i}\right]^{T}, \forall i$, and the nominal values of the states are given by: $x_{i}^{\text {nom }}=\left[\theta_{i}^{\text {nom }}, \omega_{i}^{\text {nom }}\right]^{T}, \forall i$. After any disturbance the classical swing dynamics can be easily expressed in the following perturbed system form,

$$
\Delta \dot{x}_{i}=A_{i} \Delta x_{i}+B_{i} u_{i}+G_{i} h(\Delta x) \quad \forall i
$$

This perturbed system can be classified as a linear system with a nonlinear interconnection given by

$$
h(\Delta x)=\sum_{j \neq i} P_{i j} \sin \left(\theta_{i}-\theta_{j}+\varphi_{i j}\right)-\sum_{j \neq i} P_{i j} \sin \left(\theta_{i}^{\text {nom }}-\theta_{j}^{e}+\varphi_{i j}\right)
$$

It can be easily verified that this nonlinear term can be bounded by a quadratic term in the following form: $h^{T}(\Delta x) h(\Delta x) \leq \alpha_{i}^{2} x_{i}^{T} H_{i}^{T} H_{i} x_{i}$, where $\alpha_{i}$ is a constant interconnection parameter, and $H_{i}$ is a constant matrix. The load power modulation command is defined mathematically as follows:

$$
u_{i}=K_{1 i}\left(\theta_{i}-\theta_{i}^{\text {nom }}\right)+K_{2 i}\left(\omega_{i}-\omega_{i}^{\text {nom }}\right)
$$

The optimal feedback gains $\left[K_{1 i}, K_{2 i}\right]$ obtained from the LMI optimization problem, as defined in [10], can be formulated in the following way, 


$$
\begin{aligned}
& \text { minimize } \sum_{i} \gamma_{i} \\
& \text { subject to } Y_{D}>0
\end{aligned}
$$

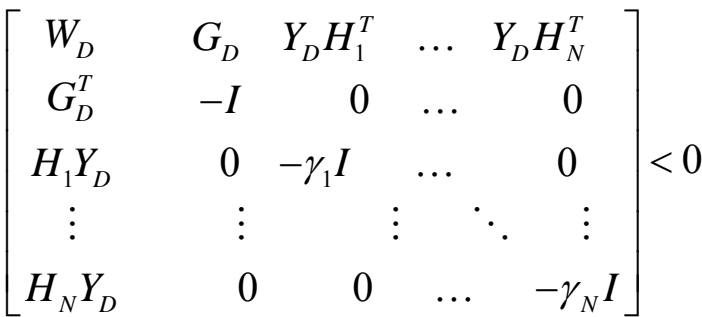

$$
\begin{aligned}
& {\left[\begin{array}{cc}
-\kappa_{L i} I & L_{i}^{T} \\
L_{i} & -I
\end{array}\right]<0 ;\left[\begin{array}{cc}
Y_{i} & I \\
I & \kappa_{Y i} I
\end{array}\right]>0}
\end{aligned}
$$

where $W_{D} \triangleq A_{D} Y_{D}+Y_{D} A_{D}^{T}+B_{D} L_{D}+L_{D}^{T} B_{D}^{T}, \quad \gamma_{i}=\frac{1}{\alpha_{i}^{2}}, \quad K_{D}=L_{D} Y_{D}^{-1}, Y_{D}=\tau P_{D}^{-1}$. In general,

$X_{D}:=\operatorname{diag}\left\{X_{1}, \ldots, X_{n}\right\}$, where $n$ is the number of buses of the system. Notice that the results of the LMI optimization process are the feedback gains which are bounded by $\kappa_{L_{i}}$ and $\kappa_{Y_{i}}$ through the inequality constraint $K_{i}^{T} K_{i}=Y_{i}^{-1} L_{i}^{T} L_{i} Y_{i}^{-1}<\kappa_{L_{i}} \kappa_{Y_{i}}^{2} I$.

When the above LMI problem is feasible, the multi-machine power system is robustly stabilized with a degree $\alpha$ by the obtained linear decentralized state feedback control law $u_{i}$. As stated before, the load power modulation command $u_{i}$ represents the amount of power to be modulated through controllable loads in order to reestablish power balance. The obtained controller is in the state feedback form, which requires the full state measurement consisting of both the voltage angles and frequency. In practice, frequency is the only local state measurement that is available at any load bus. To overcome the necessity of obtaining the measurement of the angle state by integrating frequency, further research is needed in order to implement an output feedback controller which only requires the frequency information.

After solving the above LMI problem, the obtained optimal gains $\left[K_{1 i}, K_{2 i}\right]$ are broadcasted along with the current system operating conditions, to the population of available controllable loads. In the following section, a systematic way to design load frequency response rules is developed. The strategy probabilistically turns ON and OFF each controllable load to match the desired aggregated response determined by $u_{i}$. In the proposed design, several practical aspects of controllable loads, such as compressor lockout effects, and time delays have been incorporated.

\subsection{Device-level control strategy}

When the system is subject to a contingency, a non-zero frequency deviation occurs at every bus in the system $\left(\omega_{i} \neq \omega_{i}^{n o m}\right)$. The goal of the device layer controller is to design frequency response rules for individual controllable loads so that the aggregated power change matches the desired amount $u_{i}$. For simplicity, it is assumed that the set of controllable loads is composed only by thermostatically controlled loads (TCLs). Some examples of TCLs are refrigerators, HVACs, water heaters, etc. Due to the inherent thermal energy storage, these loads can be switched ON/OFF for 30 second to 1 minute without affecting the end-use performance as discussed in [12]. The power consumption of the TCLs is assumed to be zero in the OFF state, and is a non-zero constant when in the ON state. The total controllable power 
consumption at a bus is the sum of the powers of all the controllable TCLs in the ON state on this bus. Therefore, the objective for each controllable load is to calculate its own probability of turning ON or OFF such that, the change of power consumption of controllable loads by turning ON or OFF at a given bus is equal to the load power modulation command determined at the aggregate level.

Moreover, for simplicity, the temperature dynamics of the TCL's loads as mentioned in [12] is neglected due to the fact that the temperature dynamics time scale is significantly greater than the frequency control time scale in a power system (the total period of a primary frequency response is typically less than a half minute). Additionally, in order to incorporate a more realistic behavior of the TCLs, compressor time delay constraints as mentioned in [12] were also incorporated in the load frequency controller design. This compressor time delay relay is typically installed to ensure that the compressor remains in an OFF state for a minimum amount of time, which is often referred to as a "lockout" time. During this period, a switching ON control signal will be ignored [12]. Notice that, the consideration of the lockout effect is crucial for practical implementation of load control. A simple way of capturing this behavior of incorporating the lock out effect was done using Markov chains, where each load can be in one of the following 3 states: ON, OFF or LOCKED as described in Figure 1. In particular, for simplicity the authors determined that in any simulation test, every controllable load that turns OFF actually stays LOCKED during the simulation time (40-60 seconds). This implies that each one of the controllable loads will contribute to frequency control from the demand side only once, under a given fault scenario.

A formal description of proposed load frequency control strategy is given next. Each load $k$ is associated with a discrete operating state defined by $\sigma_{k} \in\{1$ (ON), 0 (OFF), -1 (LOCKED) $\}$. The timecourse evolution of $\sigma_{k}$ is governed by a Markov Chain with transition probabilities denoted by $\operatorname{Pr}\left\{\sigma_{k}(k+\right.$ $\left.1)=j / \sigma_{k}(k)=i\right\}=: p_{i j}$. The overall transition probability matrix is given by:

$$
A=\left[\begin{array}{lll}
p_{11} & p_{01} & p_{-11} \\
p_{10} & p_{00} & p_{-10} \\
p_{1-1} & p_{0-1} & p_{-1-1}
\end{array}\right]=\left[\begin{array}{ccc}
1-\mu_{1-1} & \mu_{01} & 0 \\
0 & 1-\mu_{01} & 0 \\
\mu_{1-1} & 0 & 1
\end{array}\right]
$$

where $\mu_{i j}$ denotes the transition probability that can be modified by the load frequency controller to achieve the desired aggregated power consumption.

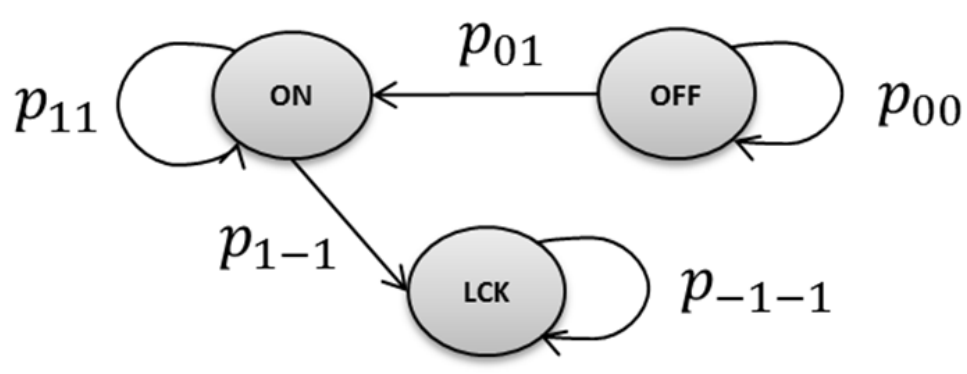

Figure 1. State transition probabilities diagram 
Notice that any load that turns off with probability $p_{1-1}=\mu_{1-1}$ becomes locked and stays locked due to the compressor time constraint. Since the lockout time is larger than the overall frequency response period, we assume that every controllable load can turn $\mathrm{ON}$ only once. The transition probability should be determined to produce the desired aggregated power change $u_{i}$. The switching probabilities are computed to match the desired power change. To find the transition probability, the aggregated load dynamics are modeled by the following Markov Chain:

$$
\begin{aligned}
& p\left(t_{k}+1\right)=A_{k}\left(t_{k}\right) p\left(t_{k}\right) \\
& y\left(t_{k}\right)=\left[C p\left(t_{k}\right)-C p(0)\right] \times P_{\text {tot }}
\end{aligned}
$$

where $p\left(t_{k}\right) \in \mathbb{R}^{3 \times 1}$ is defined as $p\left(t_{k}\right):=\left[p_{\text {on }}\left(t_{k}\right), p_{\text {off }}\left(t_{k}\right), p_{l c k}\left(t_{k}\right)\right]^{T}, C=I_{3 \times 3}$, and $y\left(t_{k}\right) \in \mathbb{R}^{3 \times 1}$ is the output (power quantity) defined as: $\mathrm{y}\left(t_{k}\right):=\left[y_{o n}\left(t_{k}\right), y_{o f f}\left(t_{k}\right), y_{l c k}\left(t_{k}\right)\right]^{T}$ and $P_{\text {tot }}$ is the available controllable power at bus $i$. From the Markov chain implementation, the probability at time $p\left(t_{k}\right)$ can be computed as: $p\left(t_{k}\right):=\prod_{i=1}^{t_{k}} A_{k}(i) \times p(0)$, then the output has the following form

$$
y\left(t_{k}\right)=\left[C\left(\prod_{i=1}^{t_{k}} A_{k}(i)\right) p(0)-C p(0)\right] \times P_{\text {tot }}
$$

Then, the goal is to approximate the response of the aggregated group of loads (power consumption) to $u_{i}$ obtained using LMI. To achieve this, the following equality must hold

$$
y_{\text {on }}\left(t_{k}\right)=u_{i}\left(\Delta \theta_{i}\left(t_{k}\right), \Delta \omega_{i}\left(t_{k}\right)\right)
$$

Moreover, it can be easily verified that $A\left(t_{k}\right)=I_{3}+B_{1} \times \mu_{1-1}\left(t_{k}\right)+B_{o} \times \mu_{01}\left(t_{k}\right)$, where $B_{1}$ and $B_{o}$ are some constant matrices Define $A(0)=I_{3}$ and $C_{1}=\left[\begin{array}{ll}1 & 0\end{array}\right]$, then the equality $y_{o n}\left(t_{k}\right)=u_{i}$ can be formulated as:

$$
\begin{aligned}
C_{1}\left[\prod_{i=0}^{t_{k}-1} A(i)\right] B_{1} p(0) \boldsymbol{\mu}_{1-1}\left(\boldsymbol{t}_{\boldsymbol{k}}\right) & +C_{1}\left[\prod_{i=0}^{t_{k}-1} A(i)\right] B_{o} p(0) \boldsymbol{\mu}_{01}\left(\boldsymbol{t}_{\boldsymbol{k}}\right)= \\
& \frac{1}{P_{\text {tot }}}\left(K_{i 1} \Delta \theta_{i}\left(t_{k}\right)+K_{i 2} \Delta \omega\left(t_{k}\right)\right)+C_{1}\left[\prod_{i=0}^{t_{k}-1} A(i)-I_{3}\right] p(0)
\end{aligned}
$$

The above equation can be easily solved by using linear programming and obtain the switching probabilities $u\left(t_{k}\right):=\left[\mu_{1-1}\left(t_{k}\right), \mu_{01}\left(t_{k}\right)\right]^{T}$ at each time $t_{k}$. If all the controllable loads turn ON/OFF according to these probabilities, the total power change will match the desired load power modulation command $u_{i}$. The inaccuracy of the Markov chain model does exist for practical applications. The robustness of our proposed design with respect to the model uncertainty has not been studied yet. 


\subsection{Simulations studies for proposed load control design}

The proposed hierarchical decentralized load frequency control strategy is validated using the IEEE 2-area, 4-generators, 13-bus power system and the IEEE 16-machine, 86-transmission line, 68-bus power system. Both systems were implemented using Power system Toolbox (PST) [18] with some modifications to incorporate load frequency response to local frequency measurement. PST is a MATLAB-based power system dynamics simulation and control design package. The advantage that it offers consists in the fact that the code is available, and thus makes it easier to access necessary system data. Moreover, supplemental MATLAB code deigned for specific functions can be added into the current toolbox version.

\subsubsection{Validation over a IEEE 13-bus 4-generators system}

The IEEE 2-area, 4-generator, 13-bus test system is shown in Figure 2. This test system contains two load buses 4 and 14. Each load bus contains controllable loads, which constitute $40 \%$ of the total power of each bus. We assume that the controllable loads reach a steady state before contingency with about $30 \%$ on, $10 \%$ locked, and $60 \%$ off. Two types of thermostatically controllable loads: refrigerators/freezers and HVACs are considered. The compressor time delay constraints are modeled for each of these loads. A symmetrical three phase short circuit fault is considered followed by a line tripping event of (3-101). The fault sequence was simulated as follows:

- In the time interval $T_{s s}=[0,5.1)[\mathrm{s}]$, the system is at pre-fault steady state

- At $t=5.1[\mathrm{~s}]$ the fault occurs

- At $t=5.15[\mathrm{~s}]$ the near end of the line is cleared

- At $t=5.2[\mathrm{~s}]$ the far end of the line is cleared

- Finally at $t>5.2[\mathrm{~s}]$, the system is in post-fault state.

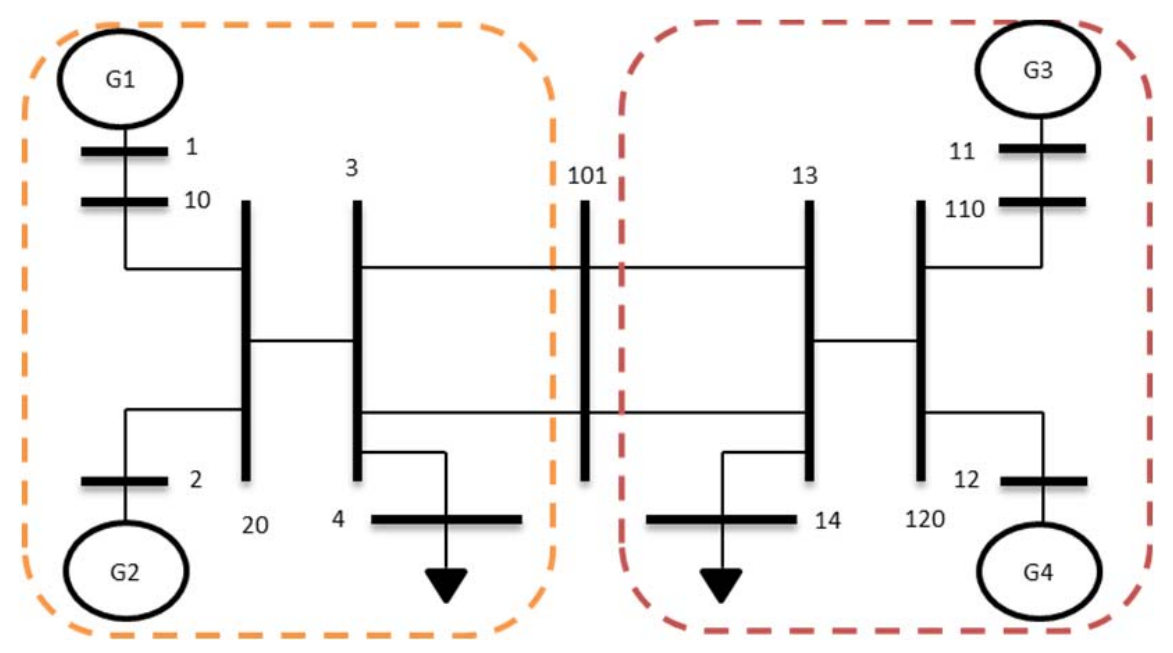

Figure 2. IEEE 2-area, 4-generators, 13-bus power system 
Response without load control: First, the response using only generation side droop control is studied. In particular, the frequency responses at the swing bus and at the load buses are shown in Figure 3. Notice that the frequency of all buses converges to a new steady-state value; this process is known as practical synchronization. In fact, the response is stable in the sense that the system is able to restore power balance, and the angle difference between buses is bounded between pre-specified limits. To restore the frequency to a nominal value, secondary control action is required. In the rest of this section, the generation-side control responses shown in Figure 3 will be used as a benchmark to evaluate the proposed load frequency control strategy. The proposed load control strategy will be demonstrated to improve both the transient and steady-state performances. Note that the generation-side AGC is not considered in the simulations. Understanding the impact of the existing AGC mechanism on the proposed load control strategy is crucial and will be important task for future research.

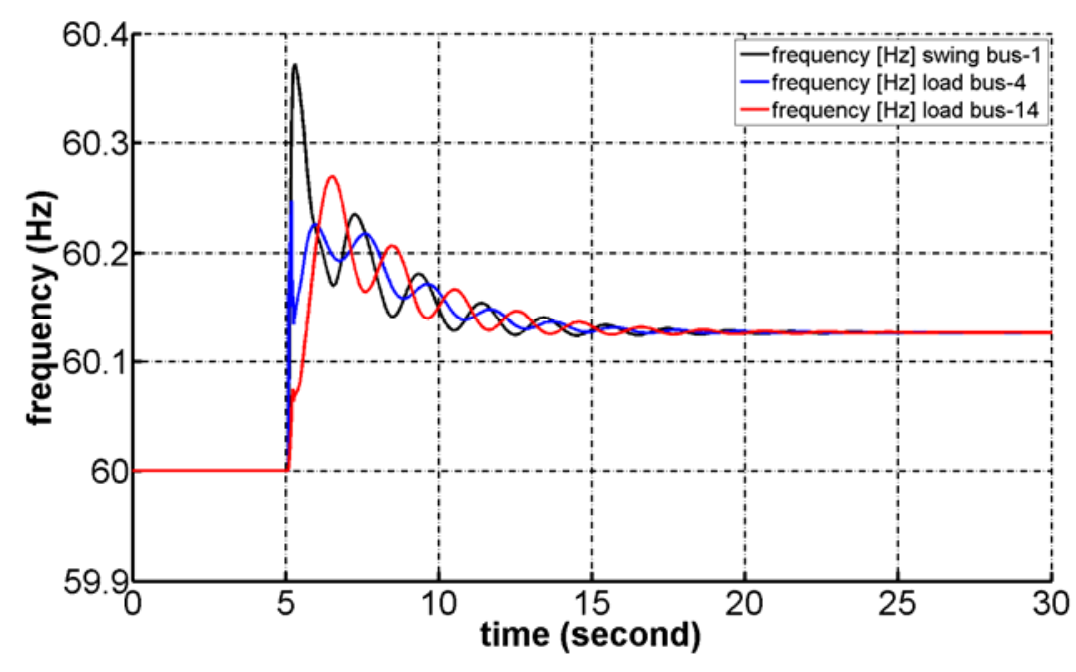

Figure 3. Frequency response with only generation side droop control

Response under heuristic load frequency controller: For comparison purposes, the load frequency control strategy proposed in [1] was implemented. According to this strategy, each load responds probabilistically to according to a predefined $\Delta f$-time profile, for which the switching probability depends not only on the instantaneous value of frequency deviation $\Delta f$, but also on the time duration the deviation has exceeded its current value. Figure 4 shows the frequency responses for two different $\Delta f$ time profiles. It can be seen with a properly tuned $\Delta f$-time profile, the frequency can be stabilized, while with a poorly tuned profile, the frequency deviation will diverge to an undesired steady-state value. A systematical way to design the local frequency response rules is not provided in [1]. A trial-and-error approach was adopted. 


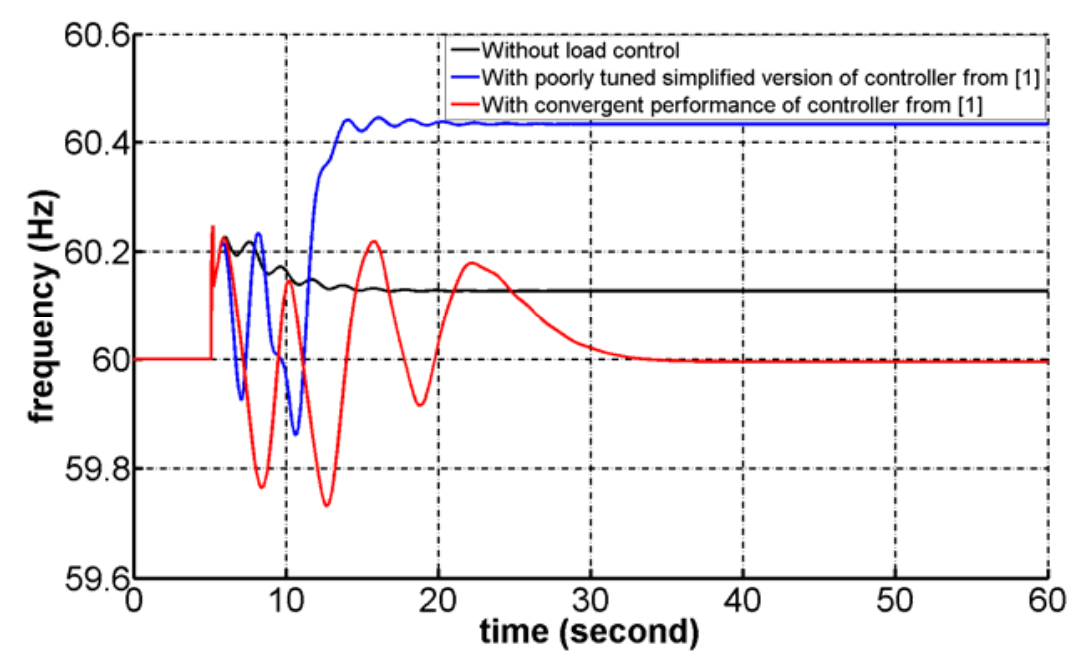

Figure 4. Frequency response at load bus-4 (simplified version of controller presented in [1])

In addition to the above frequency responses, the active power consumption at load bus 4 is also shown in Figure 5. It can be seen that the poorly tuned profile results in an unnecessarily large transient power oscillation. Due to the compressor time delay, such a large oscillation quickly locks out most of the devices, saturating the total load response. On the other hand, a properly tuned response profile is able to continuously provide a desired power change until the system reaches steady state. It is worth mentioning that the method presented in [1] may be able to achieve a good performance through extensive tuning. However, if the system conditions change, the same $\Delta f$-time profile might not be appropriate any more. Thus, a systematic design of a load control law as presented in this study has to be developed.

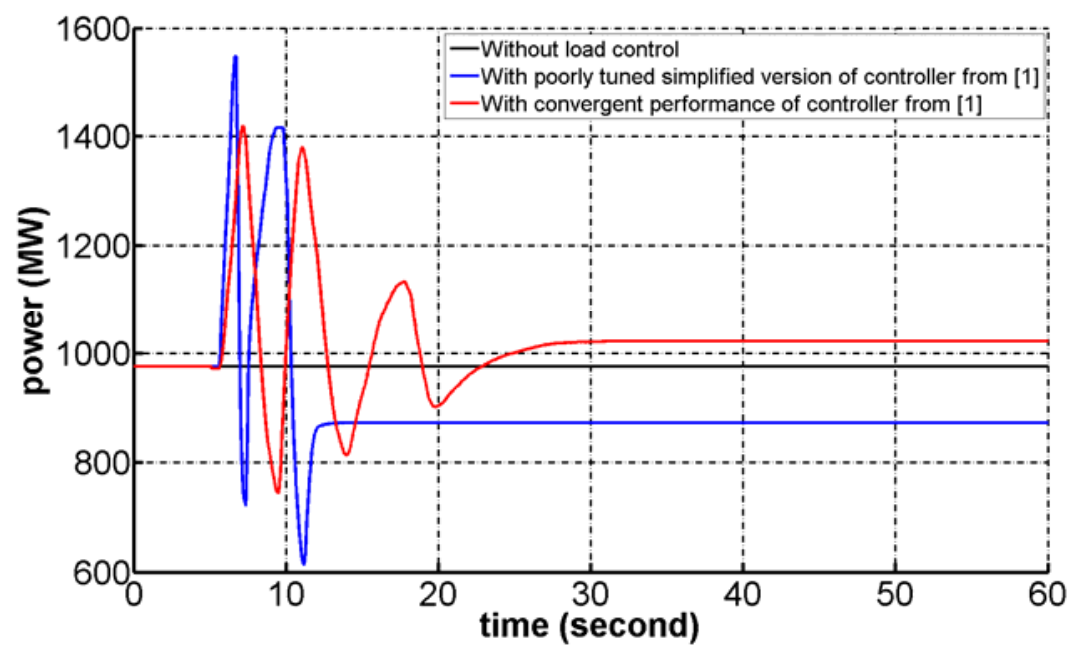

Figure 5. Active power consumption at load bus-4 (simplified version of controller presented in [1])

Responses under the proposed hierarchical distributed control design: The proposed decentralized load control law is validated under the same simulation scenario as presented above. Recall that the 
design of the proposed controller involves solving a LMI problem. If the LMI is feasible, then a linear feedback load control law can be obtained which guarantees practical synchronization and power balance across the network. The obtained control law is characterized by a control gain matrix for each load bus, which determines the desired load change in response to frequency deviations. After obtaining the load bus gains using the LMI approach, the device level controllers are designed using the Markov Chain method developed in Section 2.3. The switching probabilities for the TCLs are recomputed in a decentralized way during each simulation time step based on the load bus gain matrix and the Markov Chain model. Due to the random nature of the load response rule and the discrete sampling time step (at each $0.5 \mathrm{~s}$ the end-use device decision is updated), the desired aggregated power needs to be matched exactly by the TCL population. In the simulation results, the proposed strategy is defined to be an "ideal" feedback controller if the total controllable power on each bus is directly set to be equal to the desired power. On the other hand, the proposed strategy is referred to as a "practical" load controller if the controllable power is determined by the actual aggregated response of the TCLs that switches probabilistically based on the Markov chain approach. The simulation results, including the frequency and power responses of bus 4 and 14, are shown in Figure 6-Figure 9.

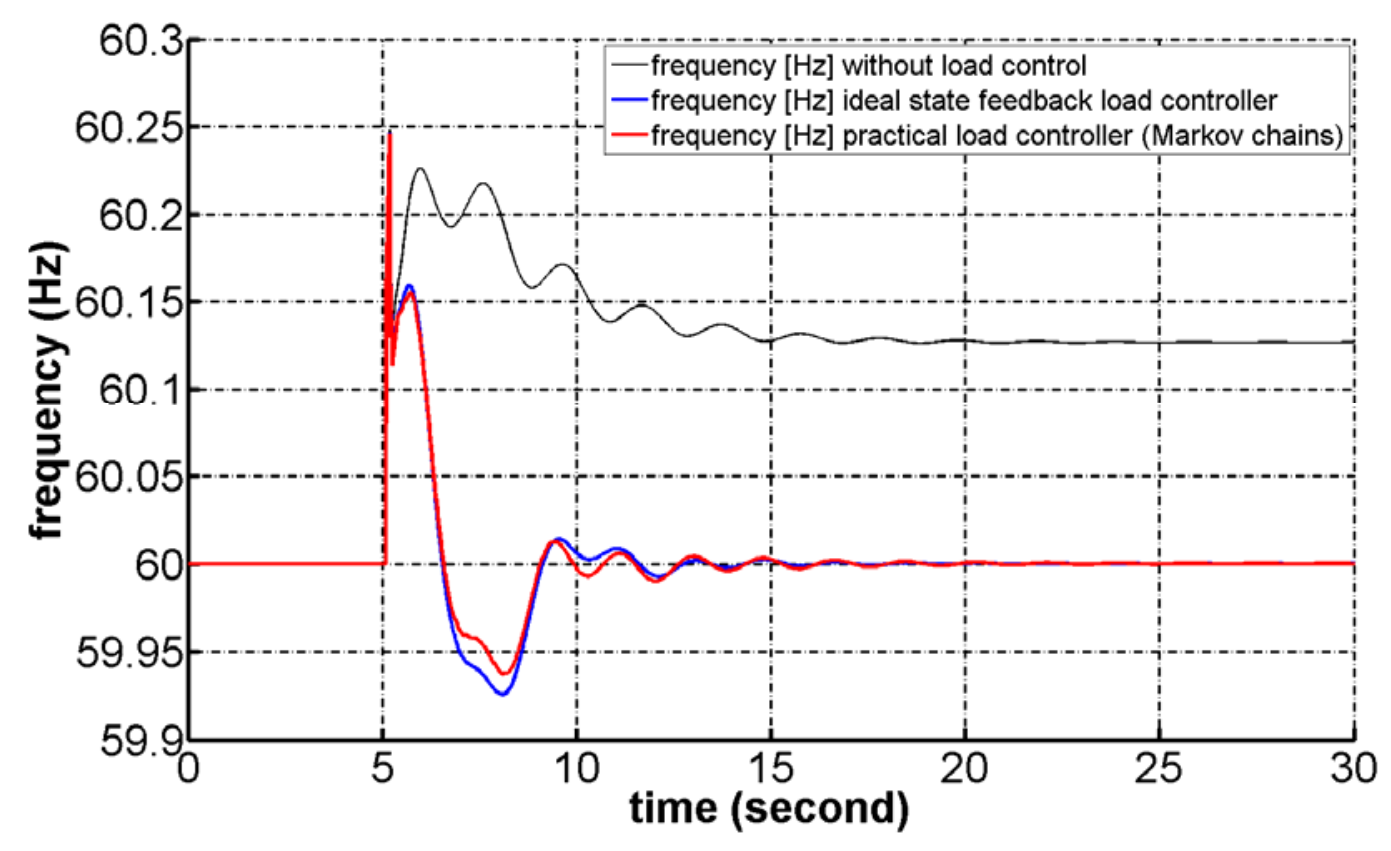

Figure 6. Frequency response at load bus 4 


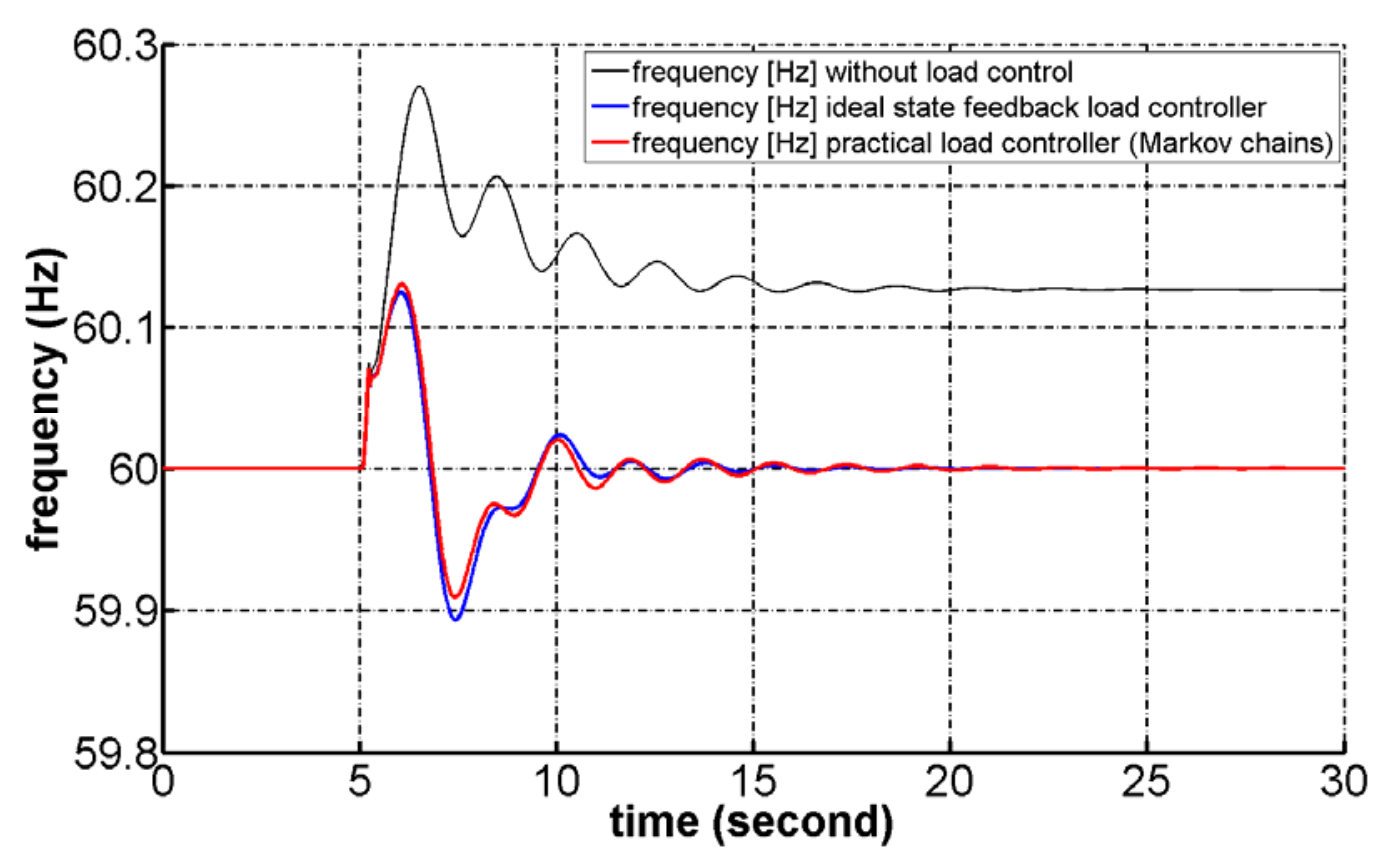

Figure 7. Frequency response at load bus 14

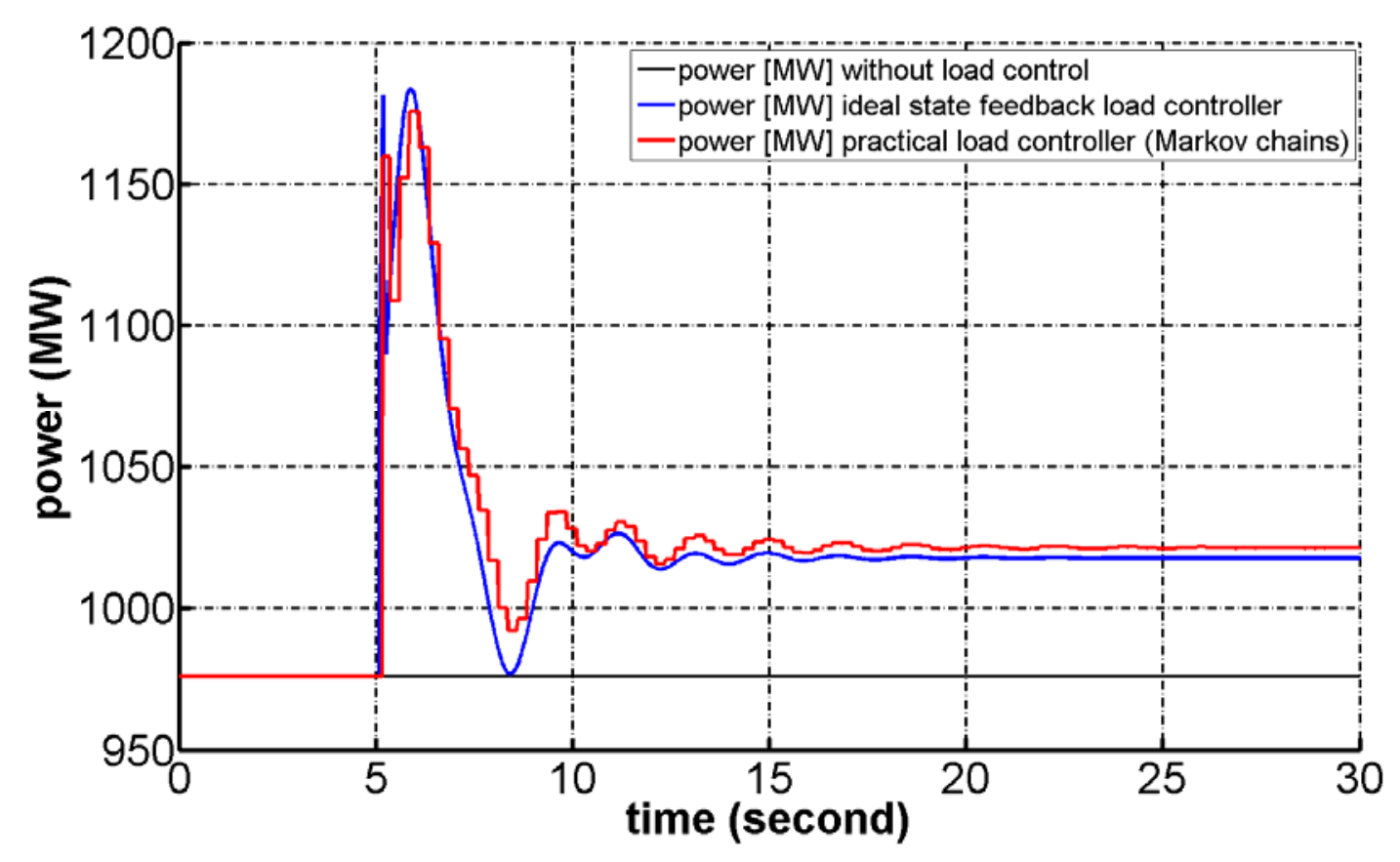

Figure 8. Active power consumption at load bus 4 


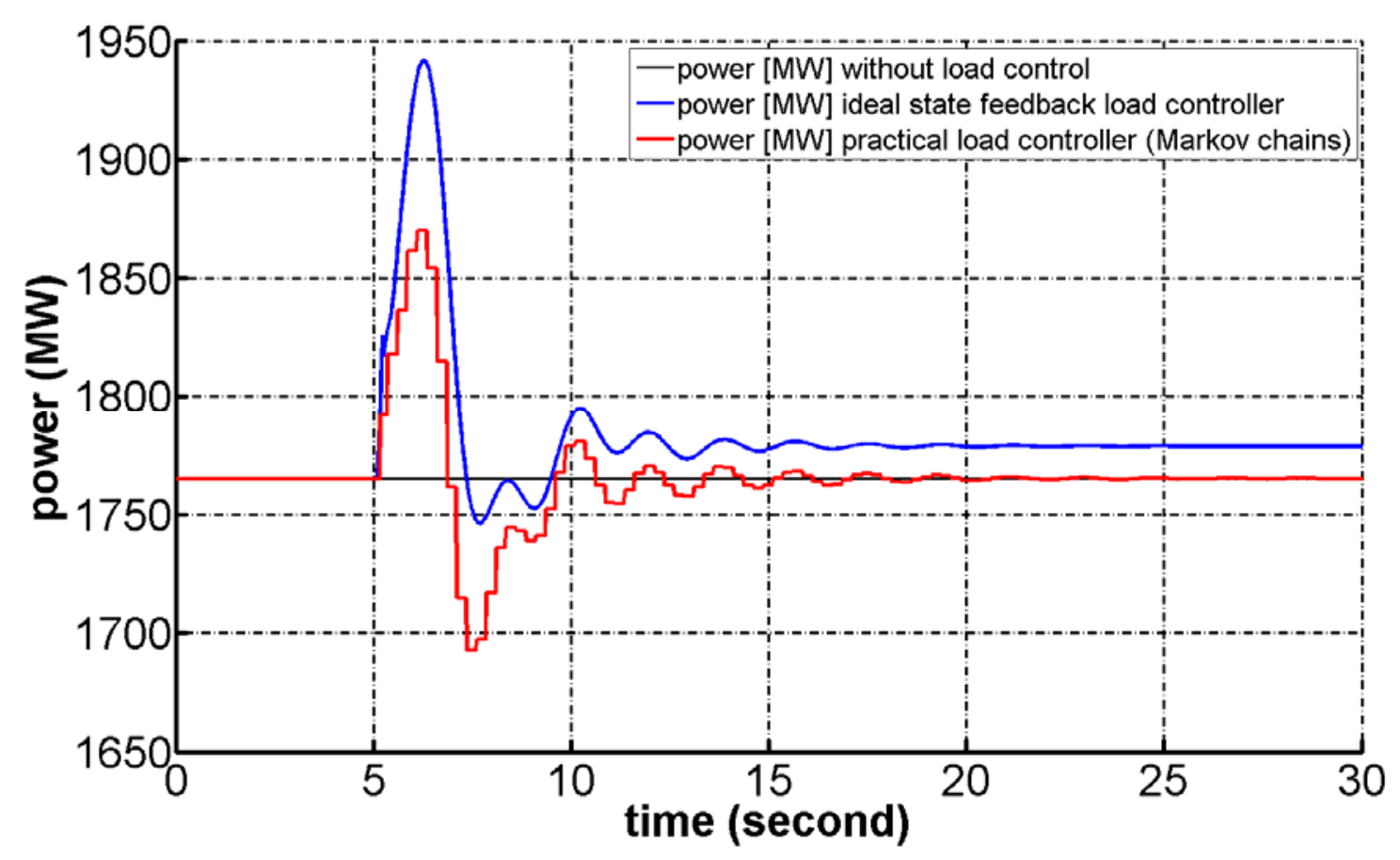

Figure 9. Active power consumption at load bus 14

These results lead to the following important observations:

- The proposed load frequency control strategy can minimize the frequency deviation under contingencies and achieve a desired steady-state system response. The response is similar to the generation-side droop control and has a better transient performance due to the LMI optimization.

- The response of the practical load controller matches well with the ideal controller. This shows a strong potential of using the proposed strategy for real load frequency control programs.

- The transient performance is much better than the heuristic controller shown in Figure 4. This is due to the fact that the LMI formulation in (5) is essentially trying to optimize the total frequency deviation during the transient subject to robust stability constraints.

- The design of the controller is done by solving the LMI problem with no heuristic tuning. This is of crucial importance because (i) it provides a systematic way to design load frequency controllers with stability guarantees, and (ii) it allows for automatic adaptation to time-varying system operating conditions.

\subsubsection{Validation over a IEEE 68-bus 16-generators system}

In order to implement the decentralized load controller in a larger system, the results are validated in IEEE 68-bus 16-generators power system as shown in Figure 10. In this model there are 24 load buses, all of which are considered to participate with controllable loads. It is also assumed that $40 \%$ of the available power on load buses is controllable. Before contingency, the population of the controllable loads is in steady state, for which $30 \%$ is $\mathrm{ON}, 10 \%$ is LOCKED, and $60 \%$ is OFF. For simulating a 
disturbance, a symmetrical three phase fault on line 36-64 followed by tripping of generator 12 was considered. The fault sequence was simulated as follows:

- In the time interval $T_{s s}=[0,5.1)[\mathrm{s}]$, the system is at pre-fault steady state

- At $t=5.1[\mathrm{~s}]$ the fault occurs

- At $t=5.15[\mathrm{~s}]$ the near end of the line is cleared

- At $t=5.2[\mathrm{~s}]$ the far end of the line is cleared

- Finally at $t>5.2[\mathrm{~s}]$, the system is at the post-fault state.

Responses under the Proposed Hierarchically Decentralized Design: The proposed load control strategy is implemented on the 68-bus system. The frequency response and the active power response on several selected buses are show in Figure 11-Figure. It can be seen from these figures that the proposed load controller can effectively restore the frequency to its nominal value. Similar conclusions regarding the advantages of the proposed strategy can be drawn as for the 13-bus case. In addition, the 68-bus simulation demonstrates initial scalability of the proposed design strategy. In future work, the effectiveness of the proposed strategy needs to be tested on large-scale WECC size system models.

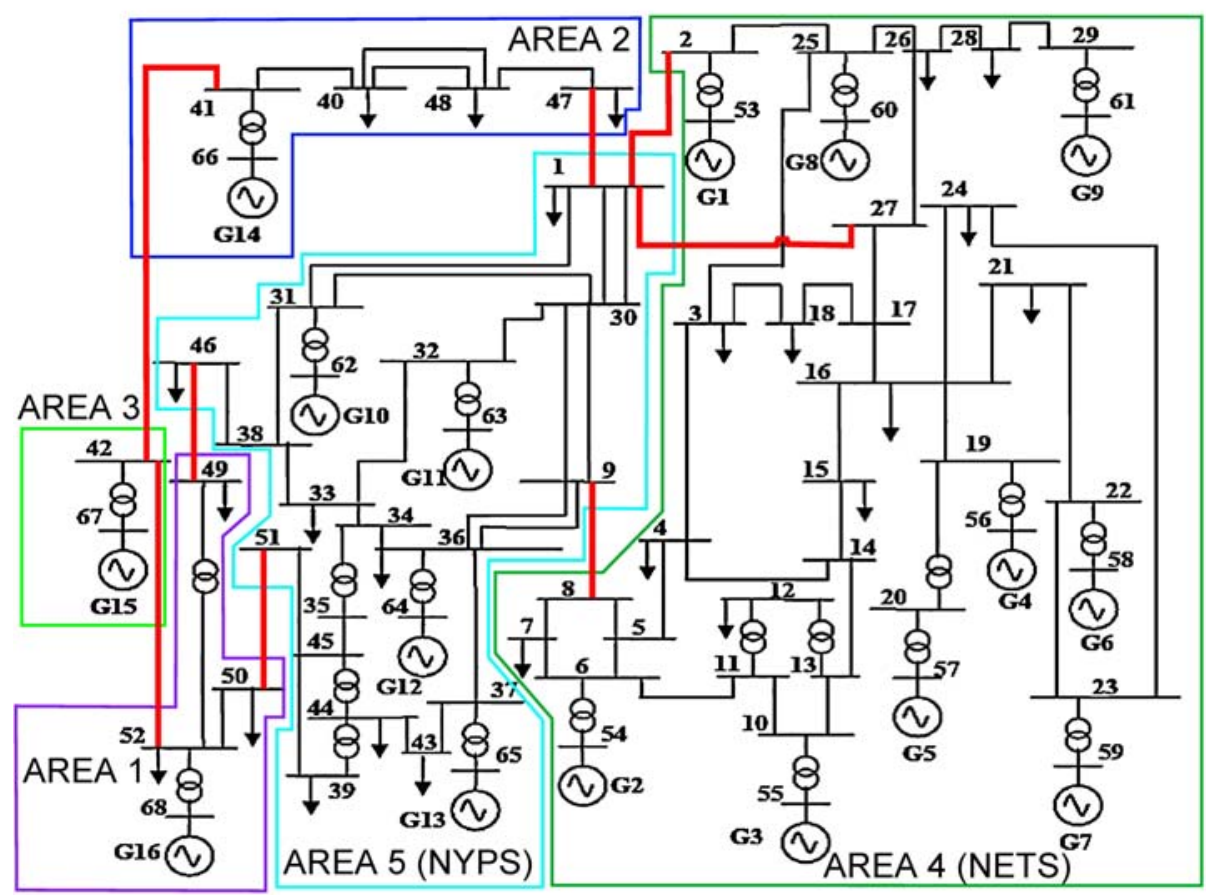

Figure 10. IEEE 16-machine, 86-transmission line, 68-bus power system (adapted from [10]) 


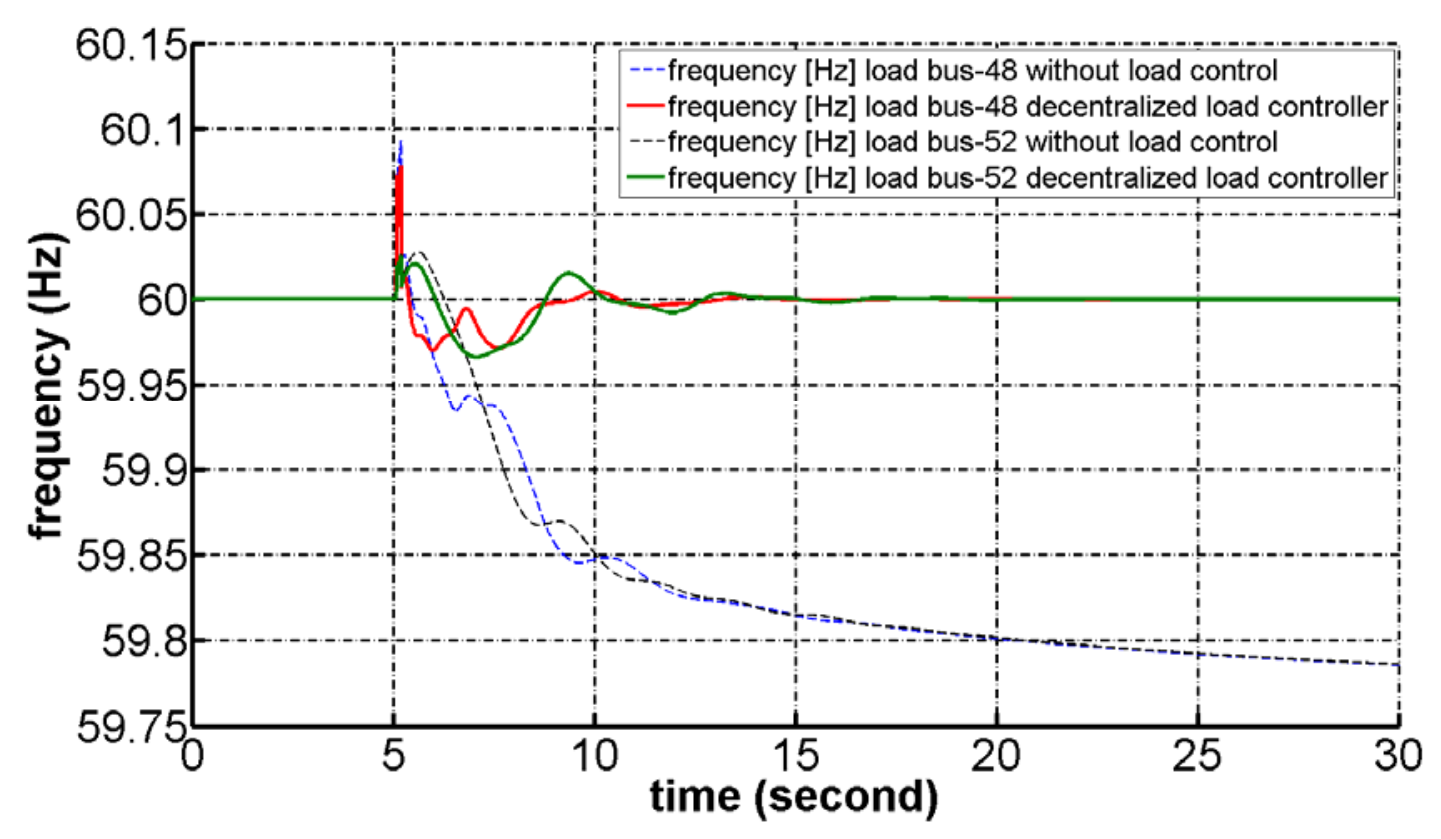

Figure 11. Frequency response at load buses 48 and 52

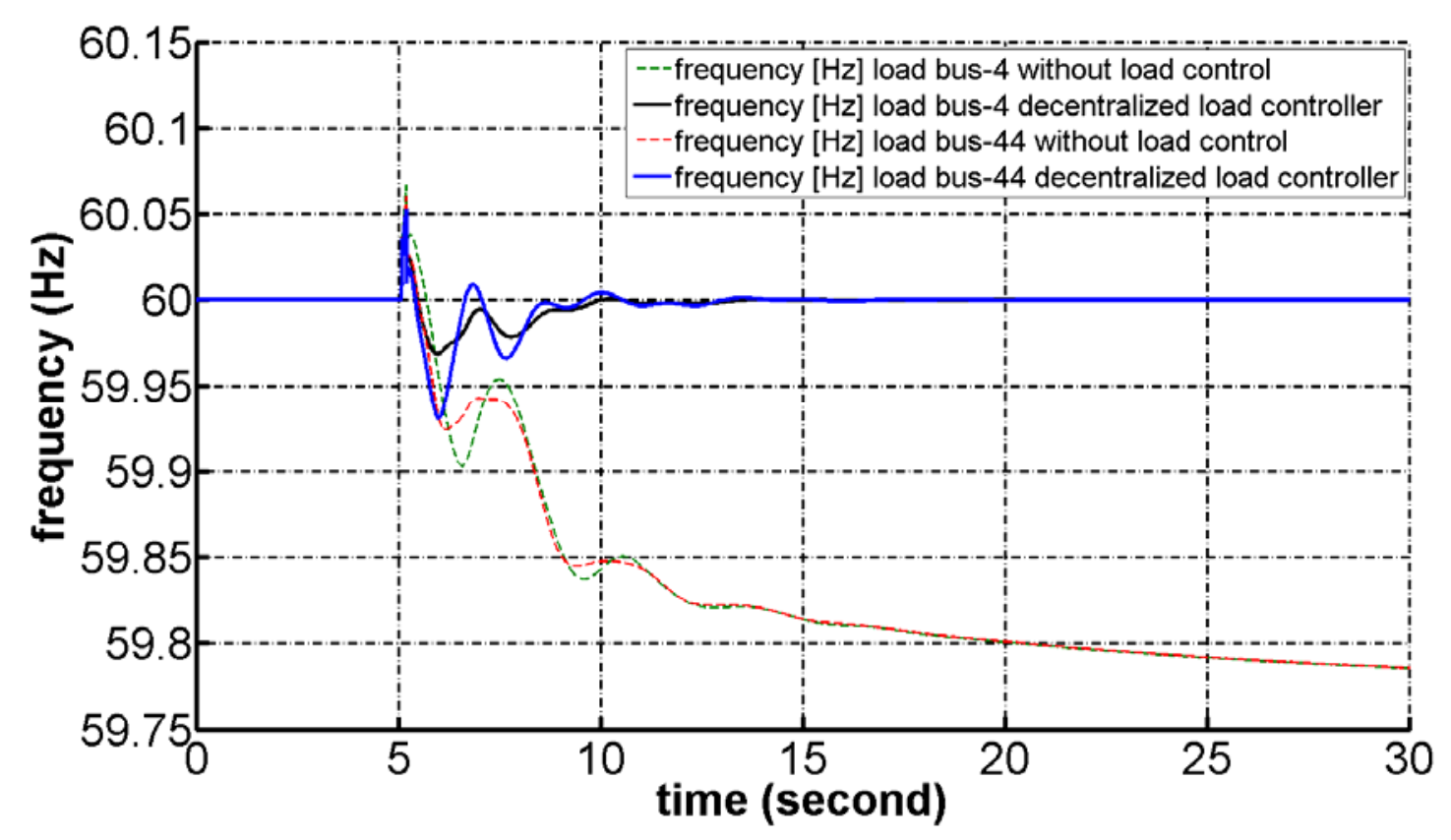

Figure 12. Frequency response at load buses 4 and 44 


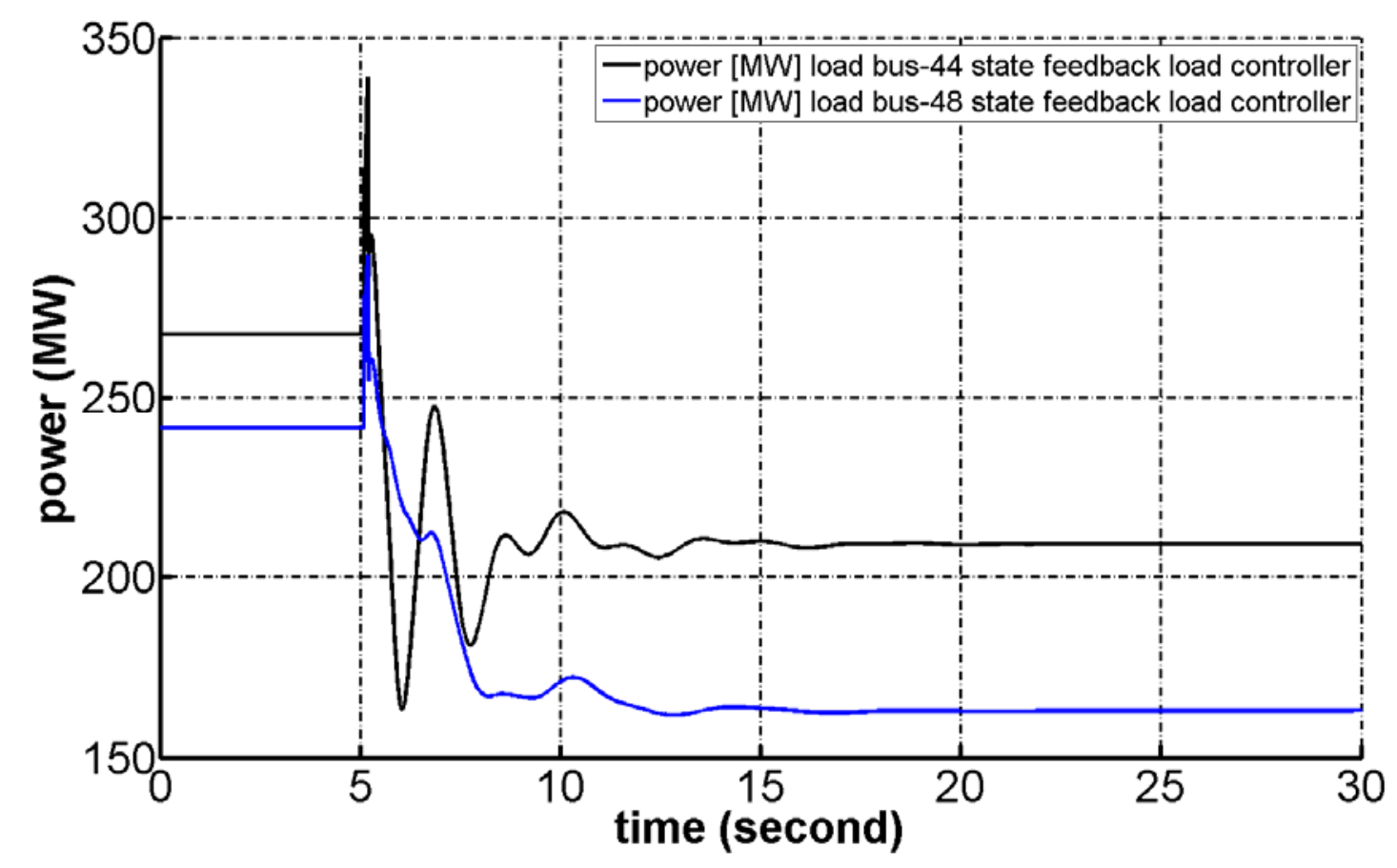

Figure 13. Active power at load buses 44 and 48

Comparison of Gain Bounds: An important parameter in the LMI formulation, defined in (5) is the bound for the droop gains. This simulation aims to gain some insight for selecting the gain bounds. To this end, two different gains are used in the LMI problem and the resulting responses are shown in Figure and Figure. The results clearly indicate that a larger gain bound will lead to a better transient response than the smaller gain bound. At the same time, a larger gain bound will also result in larger transient load power variations. Note that the simulation here is done using the ideal feedback controller. The actual achievable power variation of the population depends on the amount of available controllable loads on the corresponding bus. Therefore, in practice, the gain bound must be adjusted occasionally to respect the actual controllable loads. 


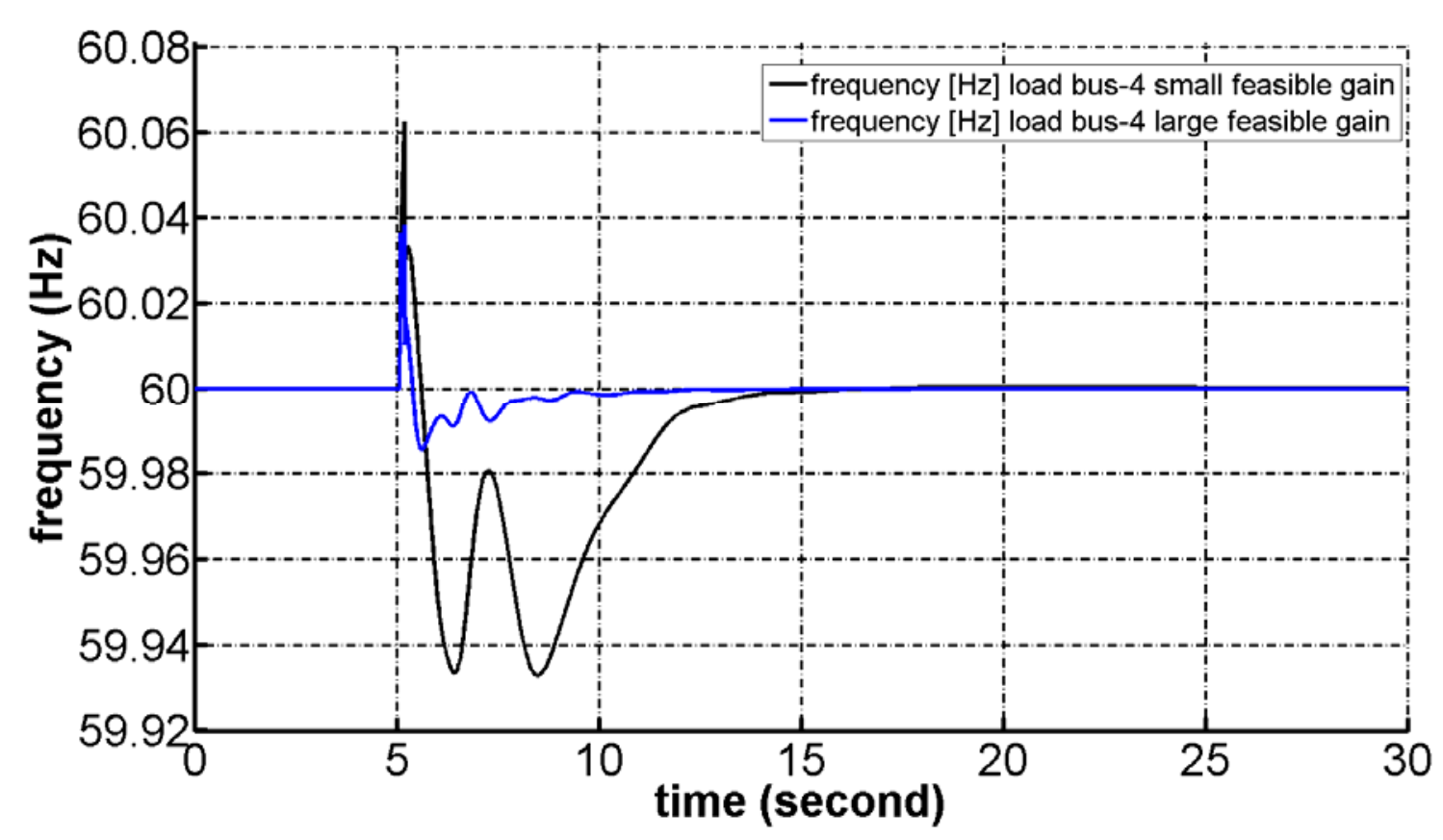

Figure 14. Frequency response at load bus 4 (Small vs. large feedback gains)

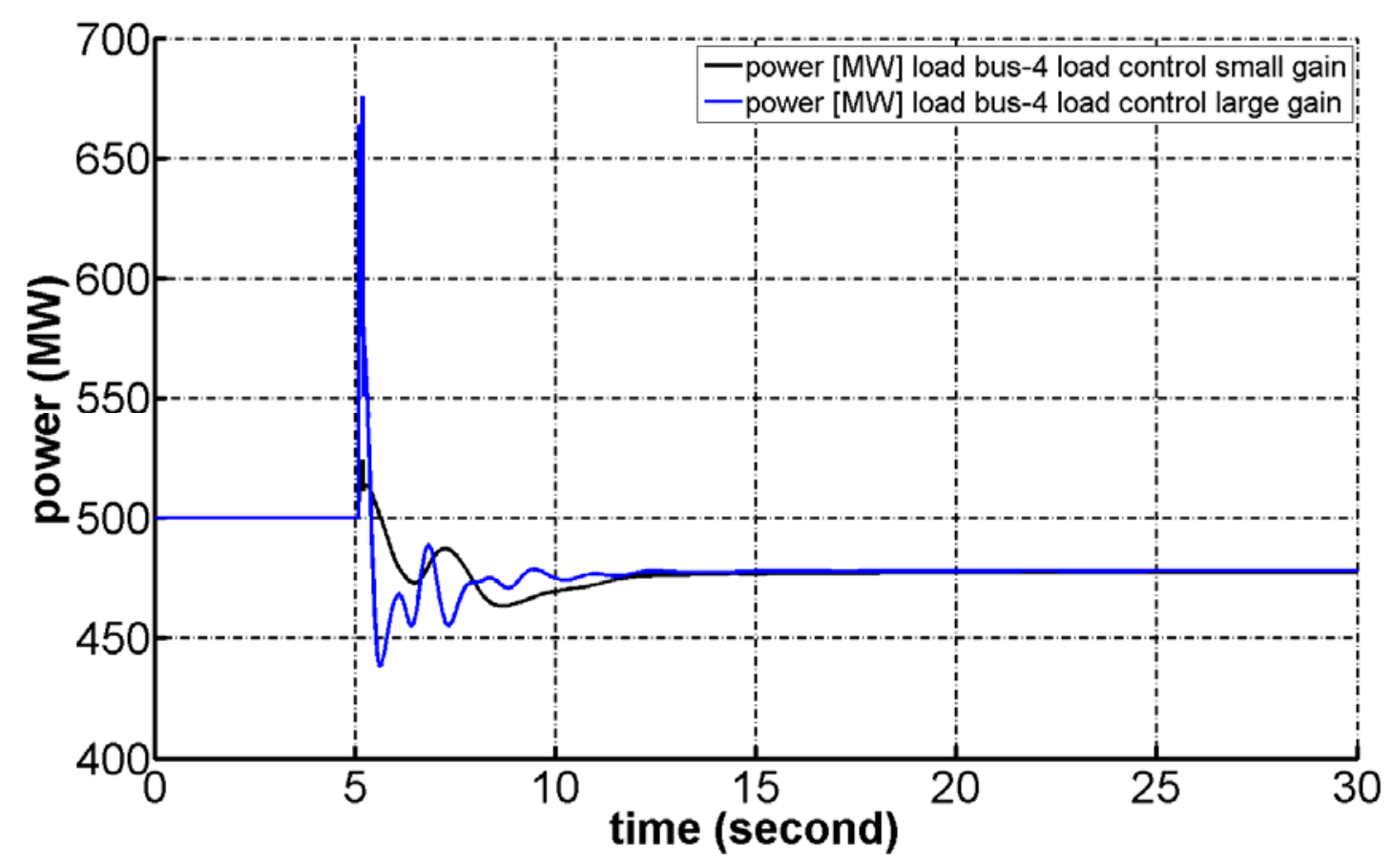

Figure 15. Active power consumption at load bus 4 (Small vs. large feedback gains) 


\subsection{Grid impact analysis of Grid Friendly ${ }^{\mathrm{TM}}$ Appliances}

The promise of autonomous, Grid Friendly response by smart appliances in the form of underfrequency load shedding was demonstrated in the GridWise Olympic Peninsula Demonstration in [19] and [20]. Grid Friendly ${ }^{\mathrm{TM}}$ Appliances GFAs controllers in the GridWise Olympic Peninsula provided autonomous under-frequency load shedding in dryers and water heaters. Each controller monitored the power grid frequency signal and requested that electrical load be shed by its appliance whenever electric power grid frequency fell below a unique and randomly chose threshold between $59.95 \mathrm{~Hz}$ and 59.985 $\mathrm{Hz}$. The controllers and their appliances responded reliably to each shallow under-frequency event, which was an average of one event per day and shed their loads for the durations of these events.

Autonomous responses are critical for many reliability purposes where there may not be time to communicate needed actions through wide-area network. Appliance and equipment manufacturers are rapidly moving toward mass production of devices with smart grid capabilities that can be leveraged for this purpose. However, utilities and balancing authorities have been hesitant to support such deployments, because the response of fleets of such devices has not been fully integrated with their control schemes for grid stability. The report presented in [21], studied the dynamic effects of populations of Grid Friendly ${ }^{\mathrm{TM}}$ water heater controllers on the power grid during under-voltage and under-frequency events. In particular, models for water heater with GFA controllers were developed using a scripting programming language and simulated in GE Positive Sequence Load Flow (PSLF), a software suite designed to study power systems in both steady-state and dynamic environments. Due to some software limitations, the project wasn't able to simulate any effect that the frequency dependent load could have on the bulk power system.

This section of the report starts with a brief description of the GFA model and control logic. The extensive simulation studies that follow are meant to illustrate the impact of a population of GFAs on the bulk power system frequency stability. The GFAs in this report are designed as demonstration units where the water heaters are individually modeled.

\subsection{GFA control logic}

The general model and control logic for the GFAs has been adopted from [21], and is schematically depicted in Figure 16. The state transition model of a GFA are described based the following four states: active, triggered, inactive (curtailed), and released. While "active", a GFA evolves based on its internal dynamics. In particular, for the water heaters with GFA controller, being active means advancing according to the electric water heater one-node model in [5]. Once an under-frequency event occurs, that is frequency drops below a lower threshold $f_{t_{-} t h}$, the GFA switches to the "triggered" state, and remains in this state for a predefined period as long as the frequency does not return to the accepted range. If the under-frequency regime persists longer than the GFA's response time $t_{d_{-} \text {off }}$, the GFA turns OFF and becomes inactive. The moment frequency passes above an upper threshold $f_{r_{-} t h}$, the GFA turns to "released" state and remains there for a certain amount of time, provided the frequency stays within tolerable limits. At the end of the turning-on time delay $t_{d_{-} \text {on }}$, the GFA is back to its normal internal behavior. 
In the subsequent study involving water heaters with GFA controllers, the turning-off time delay of each unit (the time interval between the instant the frequency drops below the threshold and the device response) is a fixed number (e.g. 0.4 seconds). This delay is actually the effect of the response time of the low-pass digital filter in charge of smoothing the frequency measurements in order to avoid reactions to unrealistic data and noise. The turning-on delay (the time interval between the moment the frequency has recovered and the moment the device is free to act according to its own dynamics) is designed to minimize the rebound effect. That means that the controlled devices will not turn on until the grid has been stabilized. The turning-on delay is randomly chosen between 2 and 3 minutes. Based on the historical data analysis described in [13], the curtailment trigger frequency for each individual water heater is set to a randomly chosen number within $59.95 \mathrm{~Hz}$ and $59.985 \mathrm{~Hz}$. Similarly, the turn-on setpoint at which the GFA is allowed to return to normal operation is randomly assigned to be between $59.995 \mathrm{~Hz}$ and $59.999 \mathrm{~Hz}$. All these frequency thresholds are uniformly distributed between the previously mentioned limits.

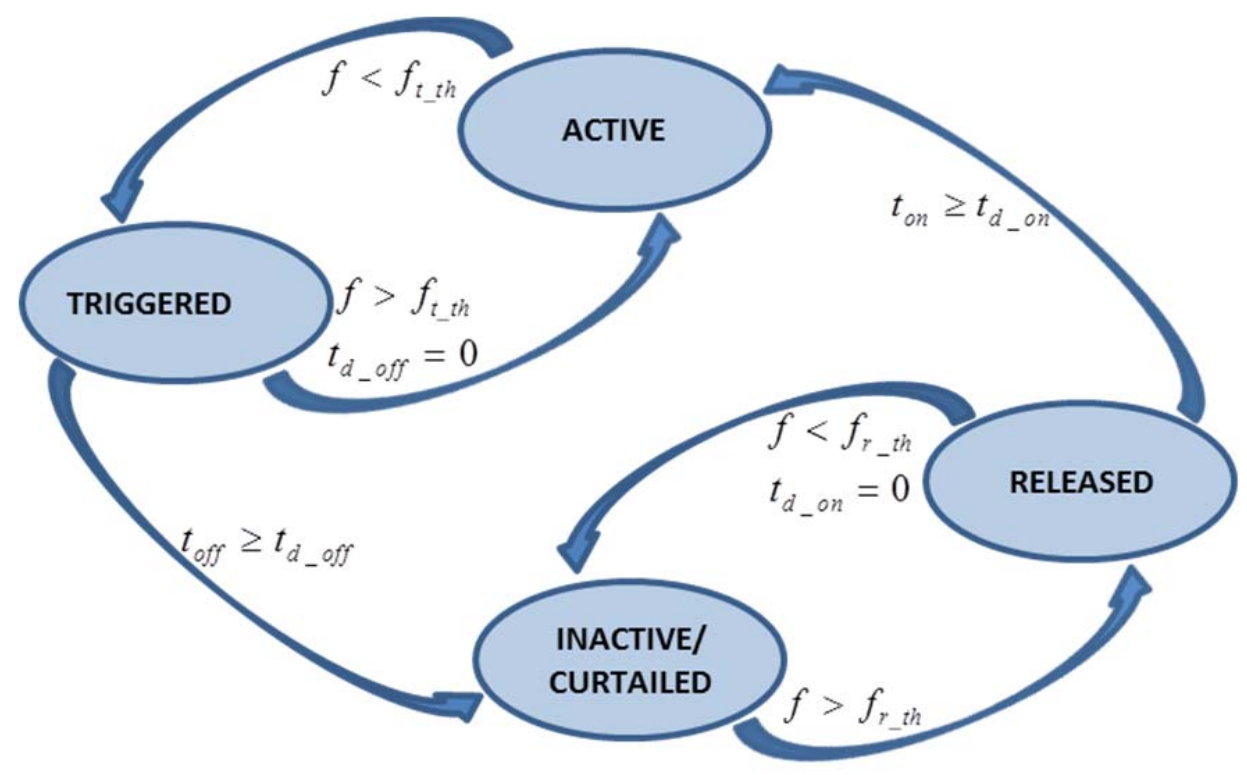

Figure 16. Underfrequency control logic for an individual water heater with GFA controller

\subsection{Description of test cases}

While the field demonstration results from the Olympic Demonstration Project were promising, the sum of the load resources controlled by the GFA controller was admittedly too small to create significant adverse impacts. Therefore, the hypotheses that a large population of such controllers could protect the system frequency, prevent actuation of substation under-frequency relays, and displace much of the need for spinning reserves remain to be definitively proven, first by simulation and then by larger field demonstrations. There have been quite a few important perspectives provided in the GFA project, which are discussed in [20]. The perspectives that are related to the grid impacts caused by GFA controllers include:

1) "One problem Grid Friendly appliance controllers face is that at times BPA does not want the devices located in the Pacific Northwest to trigger during an under-frequency event. For example, 
consider the case where the Northwest is exporting power to California and a large California generator trips off creating an under-frequency event in the Western Interconnection. In this case, Northwest generators respond by transferring momentum from Northwest generators to generators in California. If Northwest Grid Friendly devices also trip in response to an under-frequency event in California, the amount of surplus generation in the Northwest increases and more power surges down the Intertie to correct the imbalance thereby potentially increasing, rather than decreasing, system stability."

2) "A key future question is to explore the impacts of distribution of such devices (heavily weighted in the NW or PSW or evenly spread in the West) on the West-wide electrical system. Whether control springs from a central location or is distributed or autonomous is a matter of debate and concern for BPA."

Therefore, in this report, a preliminary study is performed to analyze the impacts of GFA controllers to the system frequency through detailed system simulation, aiming to provide additional perspectives to those two shown above.

\subsection{Power System Toolbox implementation of the GFA control logic}

In this work, Power System Toolbox (PST) is chosen to simulate the multi-machine power system. PST offers the advantage of being a set of open-source functions and scripts that allowed us to augment the code, in order to gain access to the necessary data. Among the many types of GFAs, the project chose to focus on electric water heaters. The one-node model specified in [5] was implemented in MATLAB, and added as a separate set of functions in PST. This model's dynamics are used to calculate the water heater power consumption based on the water temperature evolution inside the tank dictates whether the device is ON or OFF. In order to be able to employ the frequency based control logic on the water heaters, the frequency at the bus where the GFA dynamic load was connected had to be measured and broadcasted to the GFA control logic every time sample of the simulation. PST does not directly offer a bus frequency measurement. Hence, it was indirectly calculated based on the bus voltage measurements that are part of the power flow calculation. In each simulation instance, PST computes the voltage at each bus and returns it as a complex number. Hence, the voltage angle can be extracted and used to obtain the bus frequency, based on (6) and (7),

$$
\begin{gathered}
\varphi(t-\Delta t)=\int_{0}^{t-\Delta t} 2 \pi\left(f(\tau)-f_{0}\right) \mathrm{d} \tau+\varphi_{0} \\
\varphi(t)=\int_{0}^{t} 2 \pi\left(f(\tau)-f_{0}\right) \mathrm{d} \tau+\varphi_{0}
\end{gathered}
$$

as

$$
f(t)=f_{0}+\frac{\varphi(t)-\varphi(t-\Delta t)}{2 \pi \Delta t}
$$

where,

$\varphi(t)$ voltage angle at time $t$ in radians,

$\varphi_{0}$ initial voltage angle in radians,

$f(t)$ bus frequency at time $t$ in $\mathrm{Hz}$, 


$$
\begin{array}{ll}
f_{0} & \text { nominal frequency }(60 \mathrm{~Hz}), \\
\Delta t & \text { time step. }
\end{array}
$$

The frequency signal in (8) represents the input for the GFA controllers attached to the water heaters. Its values will determine whether the water heaters are curtailed or released. A fixed number of water heaters equipped with GFA controllers was considered as part of the IEEE 2-area, 4-generator, 13-bus test power system depicted in Figure 2. Both load buses 4 and 14 are set to include a certain amount of controllable loads represented by the water heaters.

\subsection{Results and discussion}

In order to examine the perspectives discussed in Section 3.2, studies are performed on the IEEE 2area, 4-generator, 13-bus test power system. All the generators are equipped with droop control. The total load in the left area is $976 \mathrm{MW}$ on bus 4, and the total load in the right area is $1765 \mathrm{MW}$ on bus 14 . Thus, the left area is exporting power to the right area. The under-frequency event is created by suddenly reducing the power generation of Generator 3 by $320 \mathrm{MW}$ at $\mathrm{t}=1.1 \mathrm{~s}$. The GFAs in these studies are selected to be water heaters, and the total power consumption of GFAs at the beginning of the simulation is around $15 \%$ of the total system load ( $400 \mathrm{MW})$. To analyze the grid impacts of the GFAs, several case studies are performed as described next.

Case I: In this case, simulation studies are performed to examine the first perspective. The response time of GFA controllers is set to be 0.4 second as specified in [20] which is the time used for hardware implementation. The system response without any GFA loads is used as the benchmark for the purpose of comparison. The system is tested first by putting all GFA loads in the left area (i.e. the Northwest), and then all in the right area (i.e. California). The simulation results are shown in Figure 17 and Figure 18. Specifically, the generator frequency responses are shown in Figure 17, and the load bus power variations are shown in Figure 18. It can be seen from Figure 17, that for the given response time of the GFA controllers and the system contingency, the steady-state system frequency deviates less from the nominal frequency in the case when GFA loads are deployed than the case without any GFA loads. Moreover, both the transient and steady-state system frequency responses are better, and the settling time is faster in the case when all the GFA loads are in the right area than the case when all the GFA loads are in the left area. The simulation results support the first perspective that there are times when GFA loads located in certain areas should not respond to under-frequency events. Thus, it is important to consider the impacts of location when deploying GFA loads to respond to the bulk system needs. 


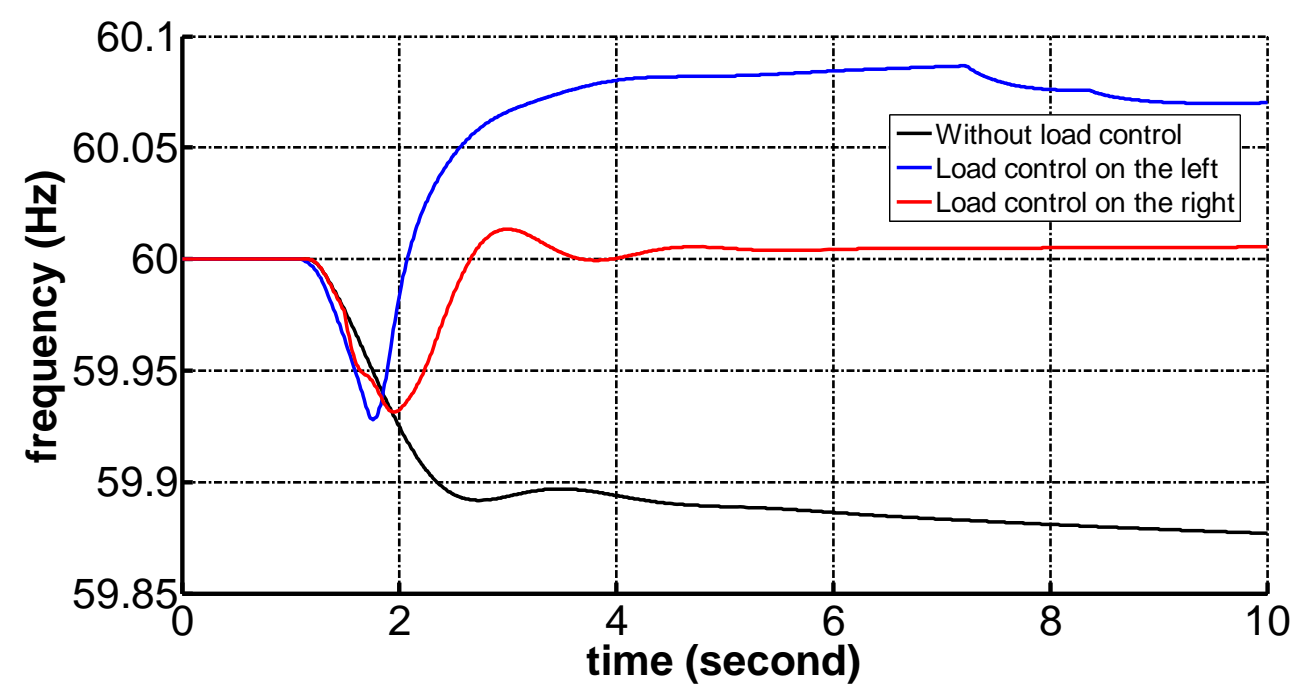

(a) Generator 2

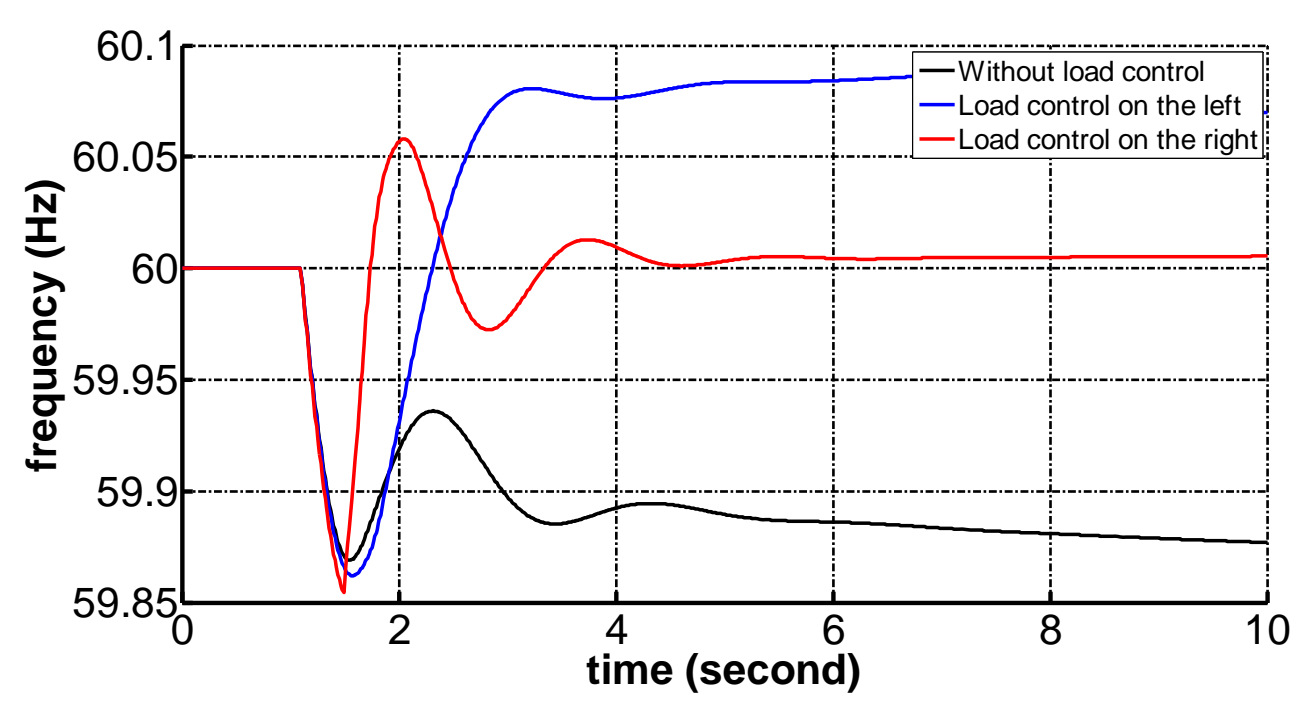

(b) Generator 4

Figure 17. Generator frequency response under GFA control with 0.4 second response time 


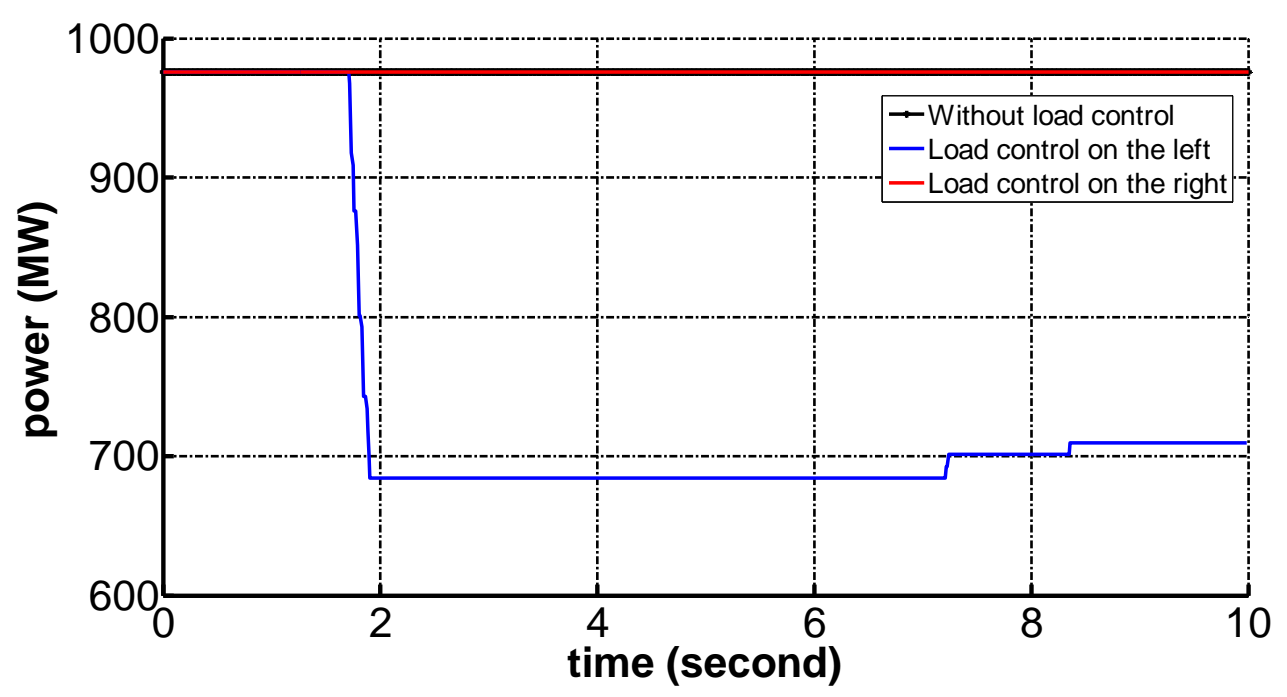

(a) Bus 4

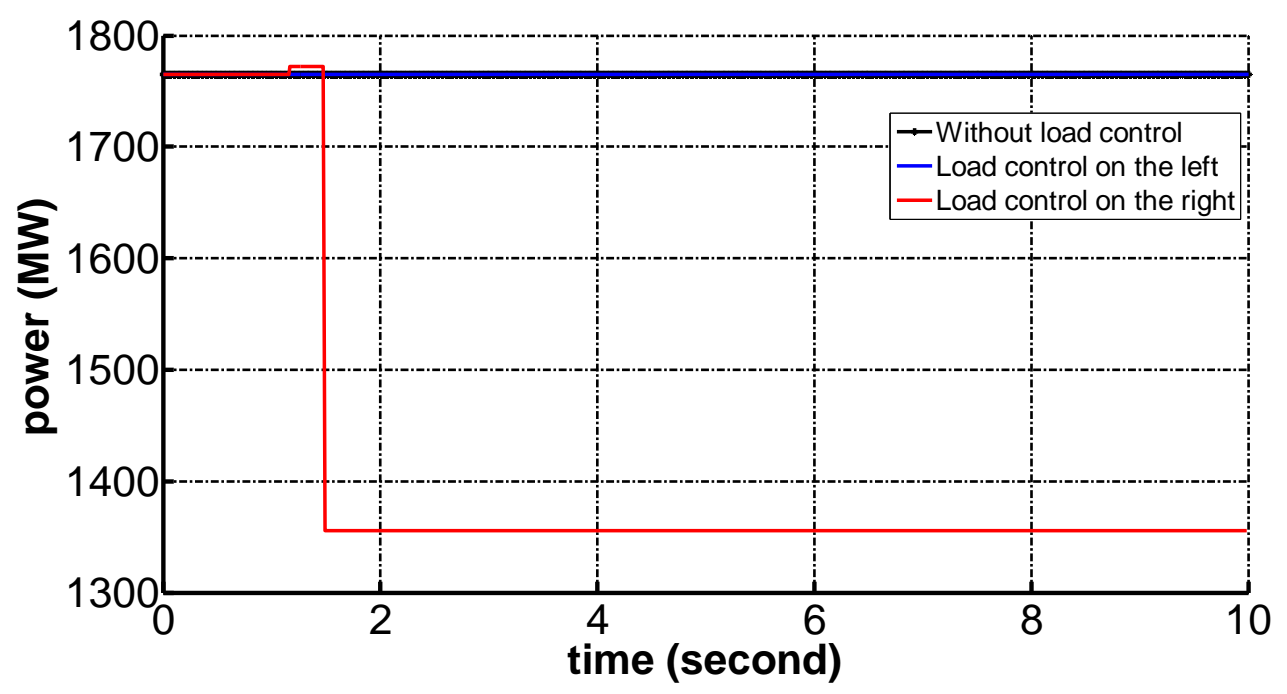

(b) Bus 14

Figure 18. Bus active power under GFA control with 0.4 second response time

Case II: In this case, the simulation studies are performed to examine how the response time of GFA controllers, one of the design parameters, affects the overall system performance. The same system tests are repeated as in Case I with three different response times for the GFA controllers, which are 0.2, 0.4 and 0.6 seconds. The simulation results are illustrated in Figure 19. It can be shown that the smaller response time give better system response. Moreover, it can be still concluded that, as far as frequency deviation and settling time are concerned, it is still better to put all the GFA loads in the right area than in the left area even for different response times. However, the problem with smaller response times is that GFA loads could respond to false under-frequency events. Thus, the appropriate selection of response time for GFA controllers needs to be carefully considered. 


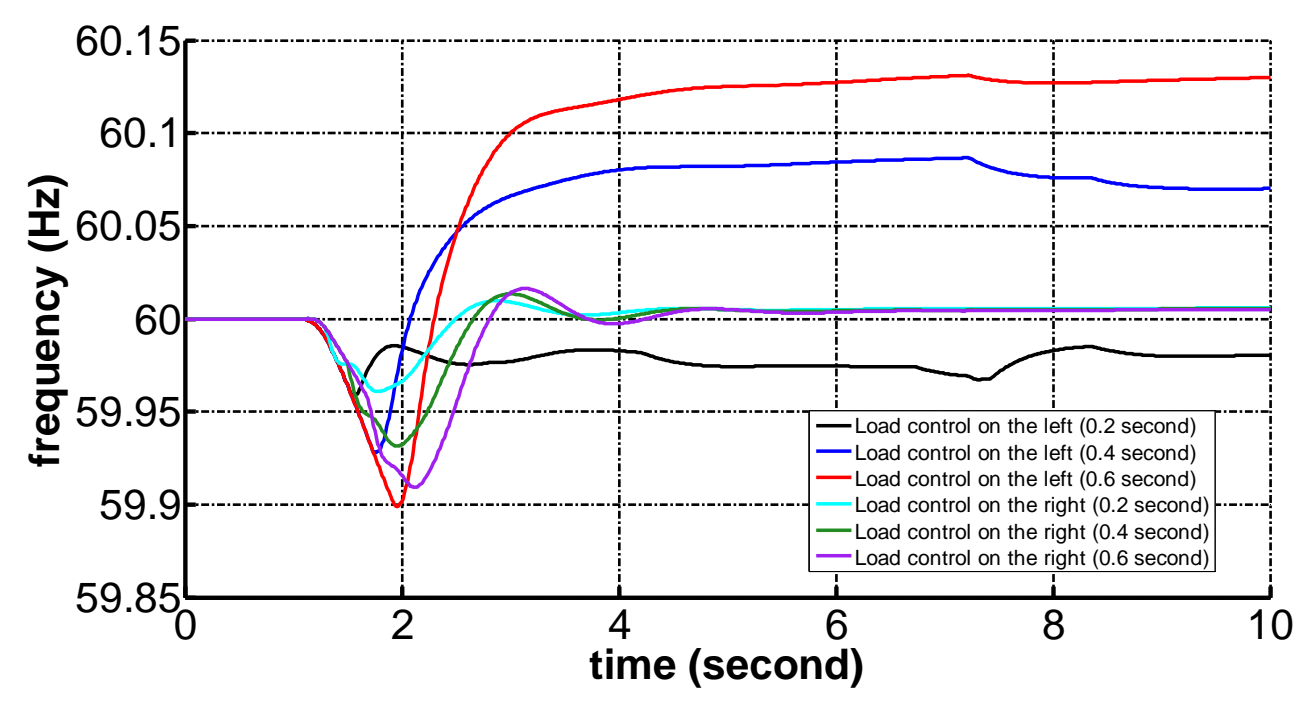

(a) Generator 2

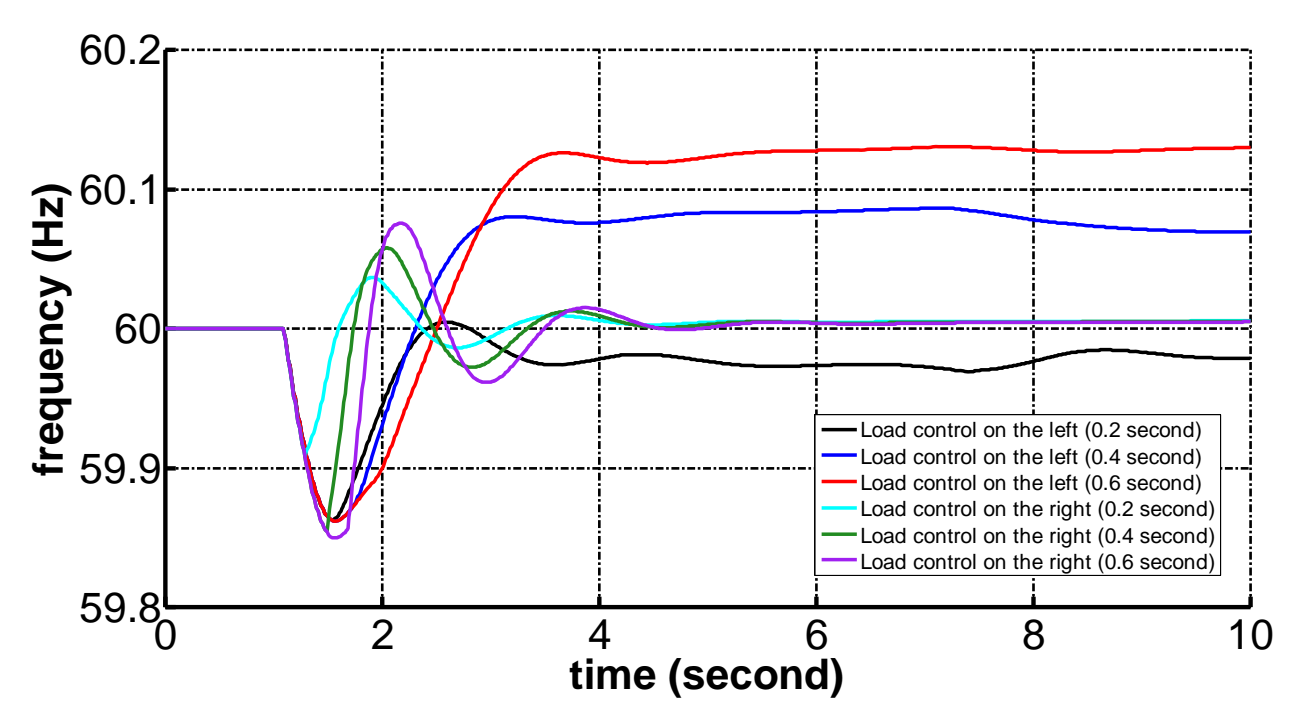

(b) Generator 4

Figure 19. Generator frequency response under GFA control with different response times.

Case III: In this case, the simulation studies are performed to examine the second perspective in Section 3.2 regarding the impacts of different distribution of GFA loads. The response time of GFA controllers is set to be 0.4 second again, and the system is tested by evenly distributing GFA loads in both the left and the right areas. The simulation results are shown in Figure 20. It can be seen that for the case when GFA loads are evenly distributed in both areas, the system response does not improve that much compared to the case when all the GFA loads are in the left area. Then the system is tested again with a different response time of GFA controllers. It can be seen from Figure 21 that when the response time is 0.2 second, the system response is the best when GFA loads are evenly distributed in both areas. However, with response time of 0.6 second, the system response is the best with GFA loads in the right 
area as shown in Figure 22. But the even distribution of GFA loads yields an improved system response as compared to the case when GFA loads are in the left area. Therefore, the distribution of GFA loads needs to be investigated together with the response time of GFA controllers because they have coupled impacts to the overall system response.

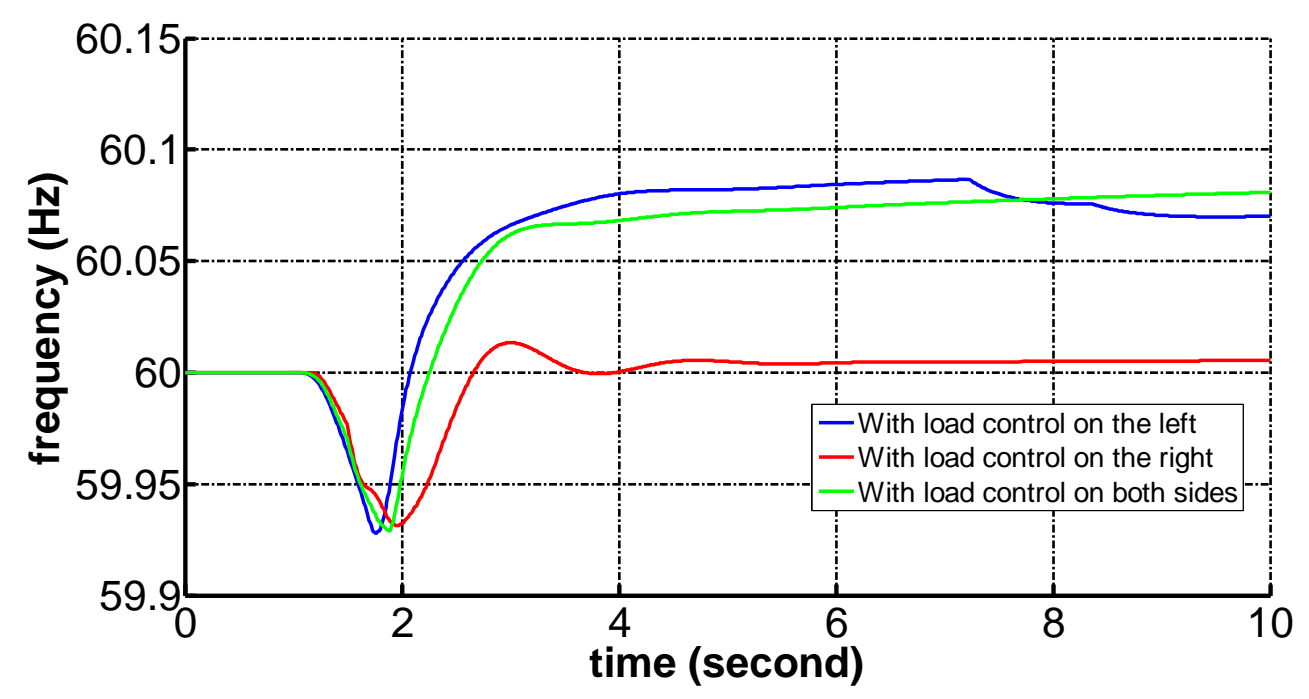

(a) Generator 2

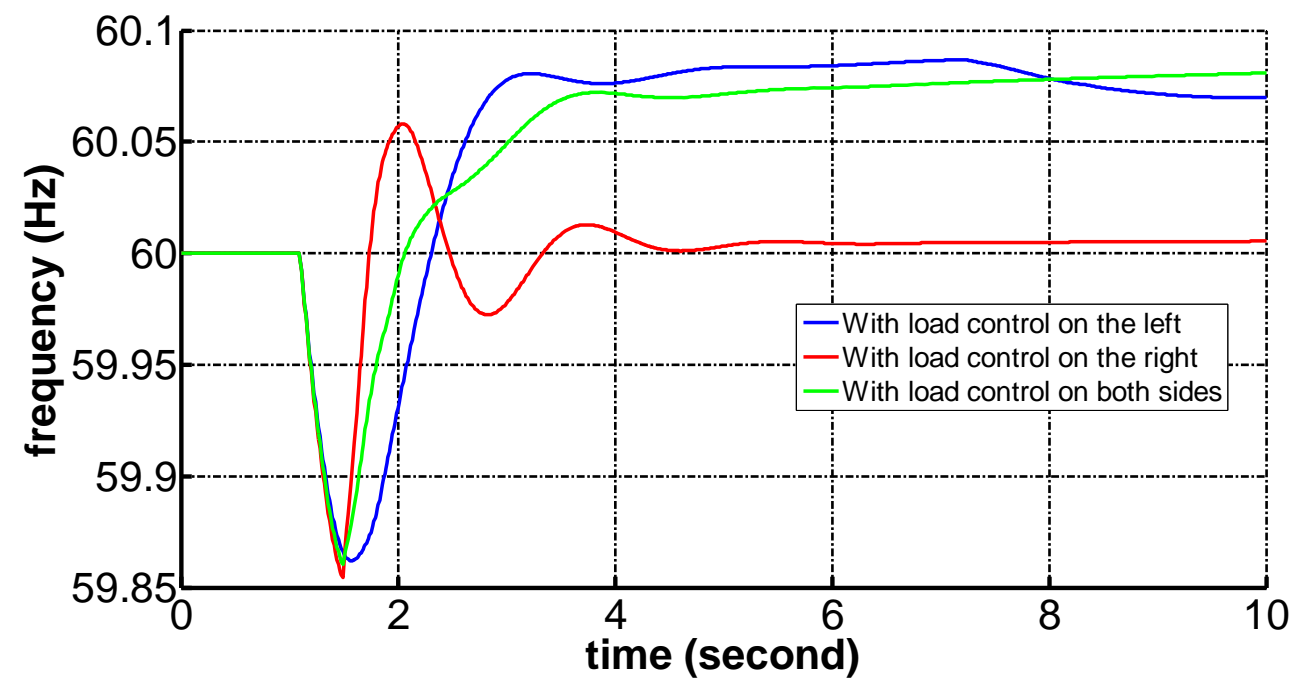

(b) Generator 4

Figure 20. Generator frequency response under GFA controller with 0.4 second response time. 


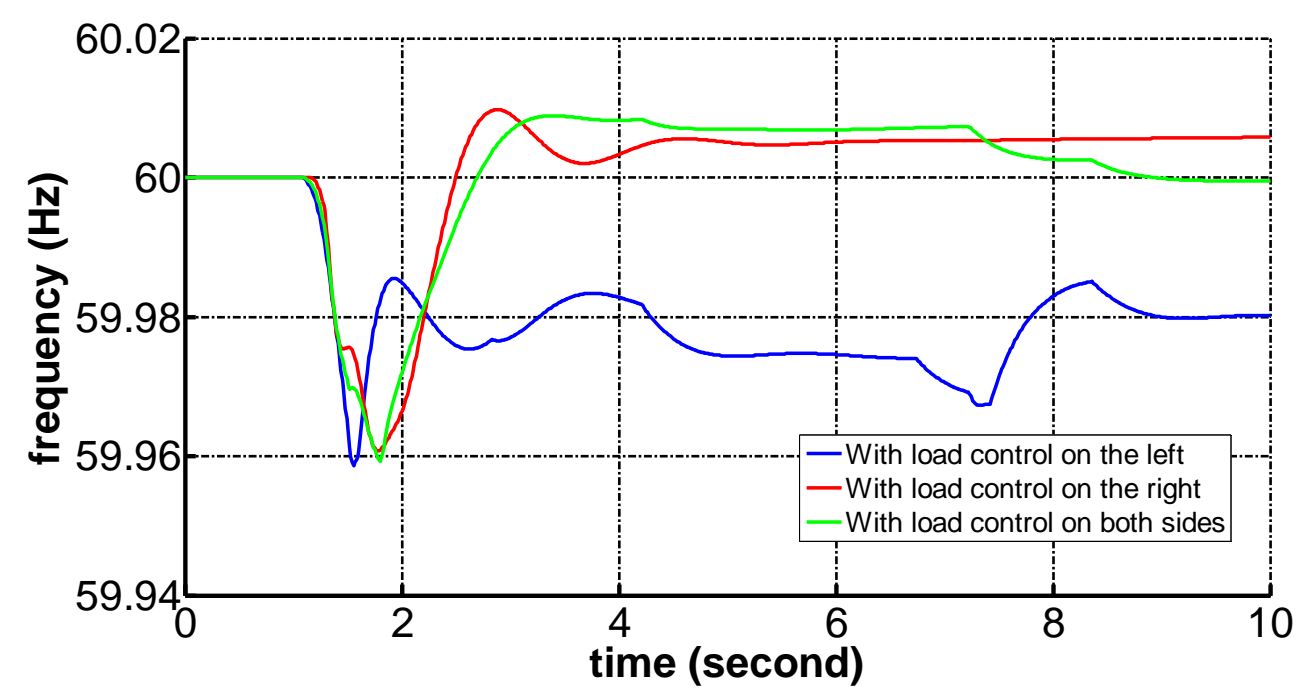

(a) Generator 2

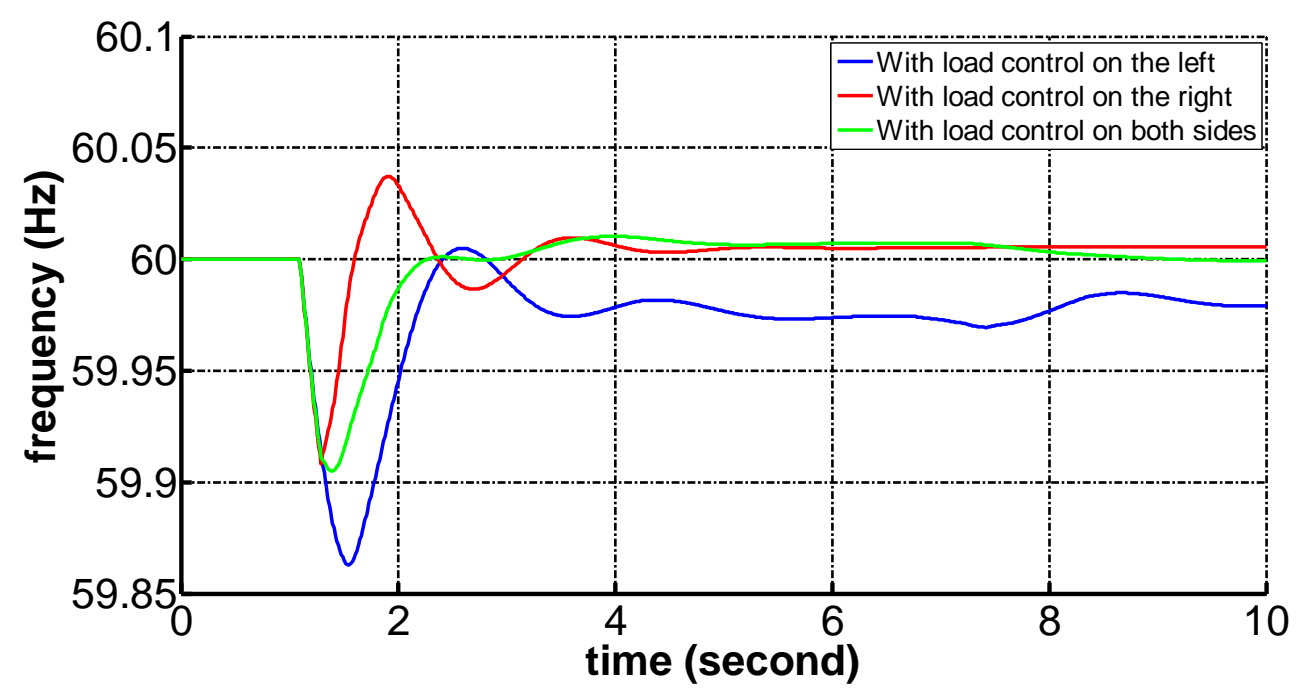

(b) Generator 4

Figure 21. Generator frequency response under GFA controller with 0.2 second response time. 


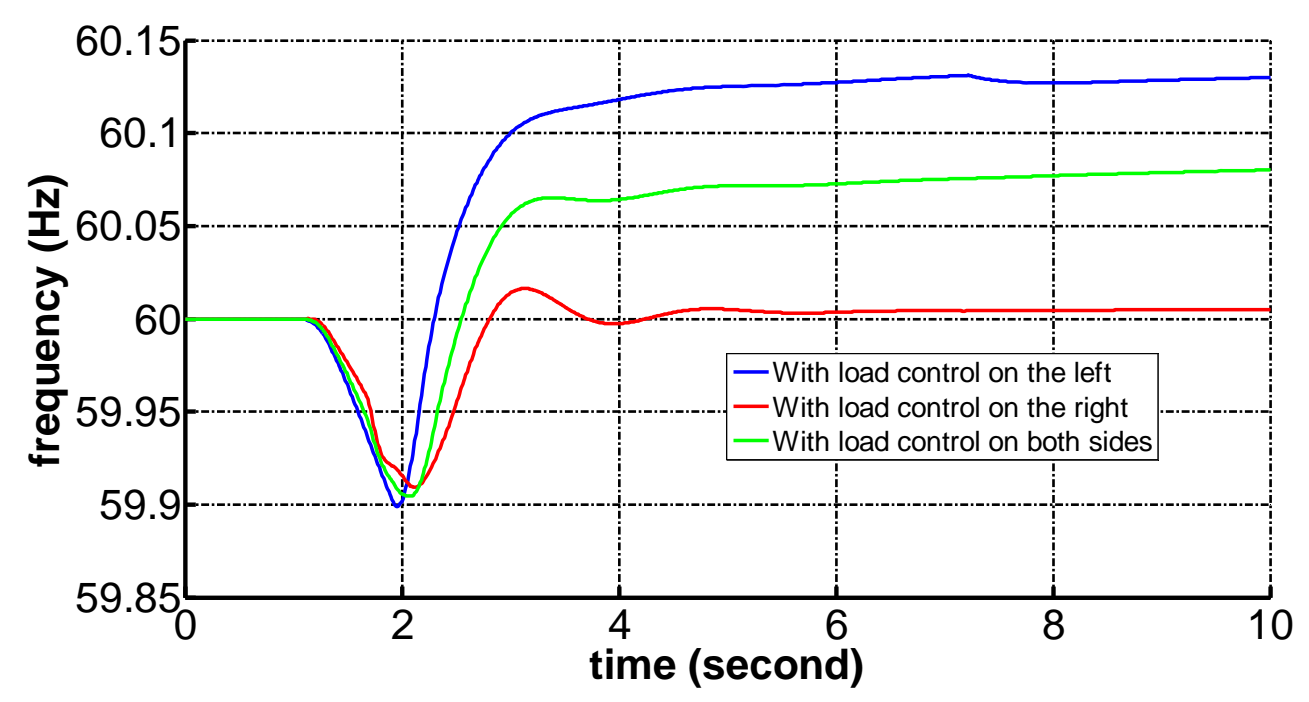

(a) Generator 2

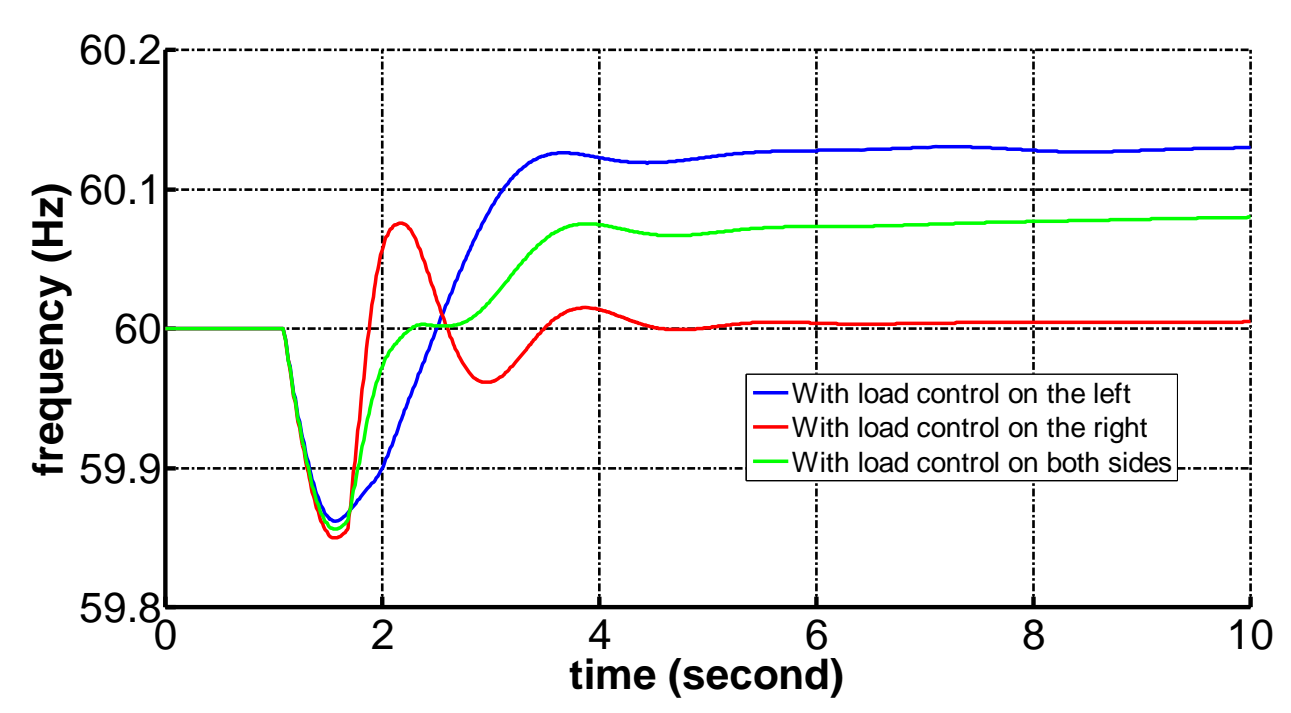

(b) Generator 4

Figure 22. Generator frequency response under GFA controller with 0.6 second response time

In the above case studies, the power penetration of GFA loads is selected to be $15 \%$. That is, the total power of GFA loads is close to the amount of generation loss that causes the under-frequency event. It is interesting to examine the system response when the power penetration of GFA loads is higher. Thus, in the following study, the penetration of GFAs is increased to be around 35\% ( $900 \mathrm{MW})$. The response time of GFA controllers is set to be 0.4 second. The simulation results are shown in Figure 23. It can be seen that the system response under higher penetration of GFA loads could potentially worsen system stability. The relationship between the amount of GFA loads and the power imbalance causing the underfrequency event should be carefully analyzed in future work to ensure the desired system response. 


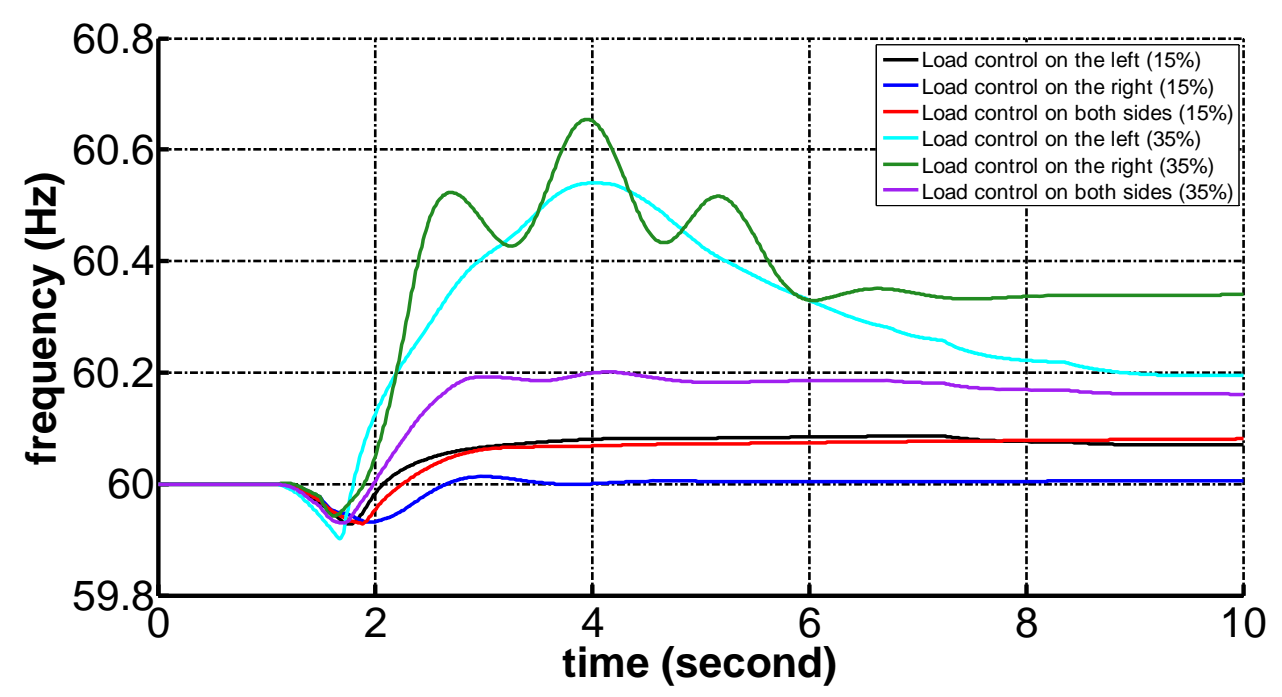

(a) Generator 2

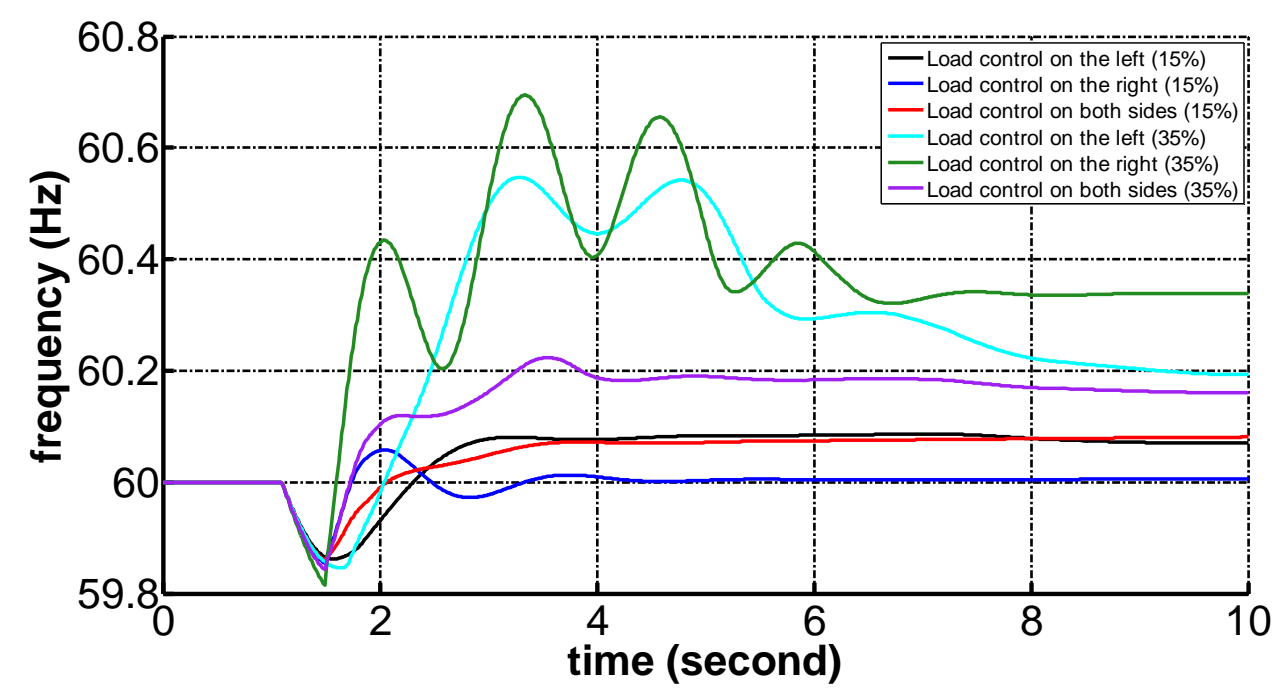

(b) Generator 4

Figure 23. Generator frequency response under different penetration of GFA loads 


\subsection{Conclusions and Future Work}

\subsection{Conclusions}

A systematic approach was proposed for a demand-side primary frequency strategy to regulate the frequency of the system to its nominal value and restore power balance for a multi-machine power system model. The load control law is designed in a hierarchically decentralized manner consisting of two interactive decision layers. In the first layer, a supervisory controller is responsible to gather system level information (e.g., power flow, system topology, generation and load forecast, available responsive loads, among others), and determine the optimal gains for the responsive loads on each bus every few (e.g. 1530) minutes. In the second layer, each decentralized load will switch ON/OFF probabilistically in real time based on local frequency measurement so that the aggregated load response under each bus matches the desired power determined by the first layer. The control gain design in the first layer is based on the decentralized robust control theory, while the load switching probabilities are designed using Markov chains.

The proposed demand-side primary frequency control strategy has several distinct features. Firstly, it systematically accounts for the interactions between the total load response and the bulk power system frequency dynamics. The decentralized-robust controller based design ensures the stability of the closedloop system over a wide-range of operating conditions. Secondly, the Markov Chain based design of local load response rules can fully respect the device constraints (such as temperature setpoint, compressor time delays of HVACs, arrival and departure of the deferrable loads, etc.), which are crucial for implementing real load control programs. Thirdly, the systematic computation of the gains every few minutes in the first control layer allows for an adaption to time-varying system operating conditions. This has not been considered in the literature but is critically important for a reliable operation of power systems. The above mentioned features make the proposed control framework both theoretically sound and practically feasible. A preliminary investigation of the grid impacts of the GFA design indicates that the location of GFAs affects system stability performance. Also, the impact of location is coupled with effects of the GFA time delay parameter.

\subsection{Future work}

Future research will mainly focus on the following extensions.

- Developing a formal relationship between the available responsive loads and the corresponding droop gain bound in the LMI formulation to reduce the conservativeness of the obtained controller

- Extend to the design framework to network preserving power system model, where a load bus needs not coincide with a generator bus

- Extend the framework to the output feedback case, where the measurements involves only frequency and its derivatives 
- Perform extensive analysis on the interactions between the proposed load frequency control strategy and the existing primary and secondary frequency controls (governor droop, AGC, etc.).

Another important next step in terms of modeling and controls of GFAs will be to improve the GFA control design such that the aggregate response mimics a generator droop like response. Furthermore, the proposed hierarchical distributed load control strategy proposed in Section 2.0 could be applied to a population of GFAs. One of the fairly restrictive assumptions in the aforementioned control strategies is the availability of state information and perfect knowledge of the model parameters. Another key component of this task will be to develop state estimators, which, in addition to estimating the states, will also be used to calibrate the reduced-order model parameters.

\section{References}

[1] Molina-Garcia, F. Bouffard and D. S. Kirschen, "Decentralized demand-side contribution to primary frequency control," IEEE Trans. On Power Systems, vol. 26, no. 1, pp. 411-419, 2001.

[2] C. Zhao, U. Topcu, and S. Low, "Frequency-based load control in power systems," in American Control Conference (ACC), 2012, 2012, pp. 4423-4430.

[3] C. Zhao, U. Topcu, and S. Low, "Swing dynamics as primal-dual algorithm for optimal load control," in Smart Grid Communications (SmartGridComm), 2012 IEEE Third International Conference on, 2012, pp. 570-575.

[4] C. Zhao, U. Topcu, and S. Low, "Fast load control with stochastic frequency measurement," IEEE Power and Energy Society General Meeting, 2012

[5] R Diao, M Elizondo, E Mayhorn, Y Zhang, "Electric water heater modeling and control strategies for demand response", Power and Energy Society General Meeting, 2012 IEEE, 2012, pp. 1-8.

[6] S. Boyd, L. El Ghaoui, E. Feron, and V. Balakrishnan, Linear Matrix Inequalities in System and Control Theory. Philadelphia, PA: SIAM, 1994.

[7] D. D. Šiljak and D. M. Stipanović, "Robust stabilization of nonlinear systems: The LMI approach," Math. Problems Eng., vol. 6, no. 5, pp.461-493, 2000.

[8] D. D. Šiljak, D. M. Stipanović, and A. I. Zecević, "Robust decentralized turbine/governor control using linear matrix inequalities," IEEE Trans. Power Syst., vol. 17, no. 3, pp. 715-722, Aug. 2002.

[9] J. Lian, L. D. Marinovici, K. Kalsi, P. Du, and M. Elizondo, "Distributed hierarchical control of multi-area power systems with improved primary frequency control," in Proc. 51st IEEE Conf. Decision and Control, Maui, HI, Dec. 2012.

[10] L. D. Marinovici, J. Lian, K. Kalsi, P. Du, and M. Elizondo, "Distributed hierarchical control architecture for transient dynamics improvement in power systems," Power Systems, IEEE Transactions on, vol. PP, no. 99, pp. 1-10, 2013.

[11] K. Kalsi, J. Lian, and S. H. Żak, "Decentralized control of multimachine power systems," in Proc. Amer. Control Conf., St. Louis, MO, Jun. 2009, pp. 2122-2127.

[12] W. Zhang, K. Kalsi, J. Fuller, M. Elizondo, and D. Chassin, "Aggregate Model for Heterogeneous Thermostatically Controlled Loads with Demand Response," IEEE PES General Meeting, San Diego, Ca, p. 1-8CA, July 2012.

[13] N Lu, DJ Hammerstrom, "Design Considerations for Frequency Responsive Grid Friendly ${ }^{\mathrm{TM}}$ Appliances", 2005/2006 IEEE/PES Transmission and Distribution Conference and Exhibition, 2006, Dallas, TX, pp. 647-652 
[14] D.S. Callaway and I. Hiskens, "Achieving Controllability of Electric Loads", Proc. of the IEEE, 99 (1), pp. 184-199, Jan. 2011.

[15] J. Mathieu, S. Koch, and D. Callaway, "State estimation and control of electric loads to manage realtime energy imbalance," Power Systems, IEEE Transactions on, vol. 28, no. 1, pp. 430-440, 2013.

[16] A. R. Bergen and D. J. Hill, "A structure preserving model for power system stability analysis," IEEE Trans. Power App. Syst., vol. PAS-100 no. 1, pp, 25-35, Jan. 1981.

[17] D. J. Hill and A. R. Bergen, "Stability analysis of multimachine power networks with linear dependent loads," IEEE Trans on Circuits and Systems, vol. CAS-31, no. 12, pp.637-645, July 1984.

[18] J. Chow and G. Rogers, "Power System Toolbox,", 3rd ed. 1991-2008 [Online]. Available: www.ecse.rpi.edu/pst/PST.html.

[19] DJ Hammerstrom, J Brous, DP Chassin, GR Horst, R Kajfasz, P Michie, TV Oliver, TA Carlon, C Eustis, OM Jarvegren, W Marek, RL Munson, and RG Pratt, "Pacific Northwest GridWise Testbed Demonstration Projects; Part II. Grid Friendly Appliance Project," PNNL-17079, 2007, Pacific Northwest National Laboratory, Richland, WA.

[20] DJ Hammerstrom, R Ambrosio, TA Carlon, JG DeSteese, GR Horst, R Kajfasz, LL Kiesling, P Michie, RG Pratt, M Yao, J Brous, DP Chassin, RT Guttromson, OM Jarvegren, S Katipamula, NT Le, TV Oliver, and SE Thompson, "Pacific Northwest GridWise Testbed Demonstration Projects; PartA.

[21] N Lu, DJ Hammerstrom, and S Patrick, "Grid Friendly ${ }^{\mathrm{TM}}$ Device Model Development and Simulation”, PNNL-18998, 2009, Pacific Northwest National Laboratory, Richland, WA 



\section{Distribution}

1 Phil Overholt Department of Energy, OE 1000 Independence Ave., SW Routing OE-10

Washington, DC 20585
8 Local Distribution

Pacific Northwest National Laboratory

Karanjit Kalsi

Jianming Lian

(PDF)

Laurentiu Marinovici

(PDF)

Jeff Dagle

Robert Pratt

Jason Fuller

Don Hammerstrom

David Chassin
(PDF)

(PDF)

(PDF)

(PDF)

(PDF)

(PDF)

1 Wei Zhang 404 Dreese Labs, 2015 Neil Ave.,

Columbus, OH, 43210

(PDF copy) 


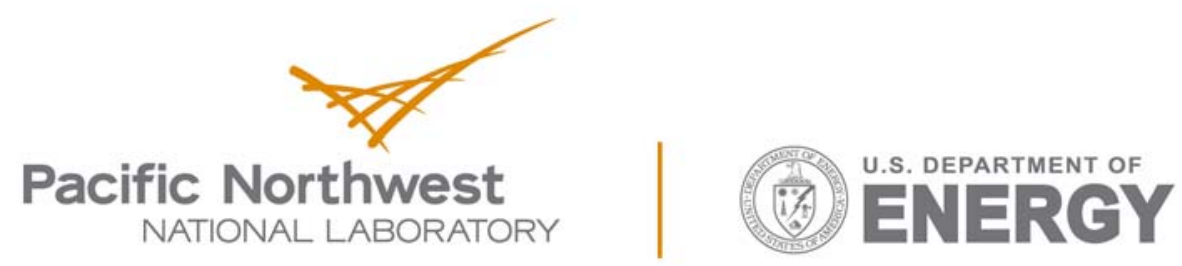

Proudly Operated by Battelle Since 1965

902 Battelle Boulevard

P.O. Box 999

Richland, WA 99352

1-888-375-PNNL (7665)

www.pnnl.gov 